\title{
Synthesis of the C3-C19 Segment of Phorboxazole B
}

Justin P. Vitale, Scott A. Wolckenhauer, Nga M. Do and

Scott D. Rychnovsky*

Department of Chemistry, 516 Rowland Hall, University of California-Irvine,

Irvine CA, 92697-2025

srychnov@uci.edu

\section{Table of Contents}

GENERAL EXPERIMENTAL DETAILS.............................................................................................................. 1

FIRST ROUTE TO PRODUCT 23........................................................................................................................ 3

SYNTHESIS OF PRODUCT 24 USING A TMSBR-PROMOTED CYCLIZATION........................... 17

SYNTHESIS OF PRODUCT 13 USING A TFA-PROMOTED CYCLIZATION ................................. 25

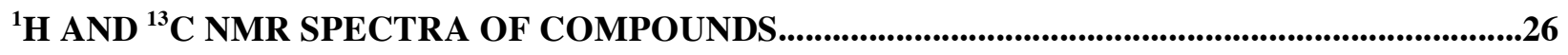

\section{General Experimental Details}

Unless otherwise stated, all reactions were carried out under an inert atmosphere of argon or nitrogen using standard syringe/septa techniques. All glassware was flame- or oven-dried and cooled under an inert atmosphere of argon or nitrogen unless otherwise stated. All commercially available reagents were used as received with the following exceptions. Tetrahydrofuran, diethyl ether, dichloromethane, hexanes and toluene were degassed with argon and dried by vacuum filtration through activated alumina according to the procedure described by Grubbs. ${ }^{1}$ Pyridine, triethylamine and $\mathrm{BF}_{3} \cdot \mathrm{OEt}_{2}$ were distilled from $\mathrm{CaH}_{2}$ under argon at atmospheric pressure. Acetic anhydride was dried over $\mathrm{P}_{2} \mathrm{O}_{5}$ decanted from the solid material and distilled from anhydrous $\mathrm{K}_{2} \mathrm{CO}_{3}$ under argon at atmospheric pressure. $3 \AA$ and $4 \AA$ molecular sieves were

\footnotetext{
${ }^{1}$ Pangborn, A. B.; Giardello, M. A.; Grubbs, R. H.; Rosen, R. K.; Timmers, F. J. Organometallics 1996, 15, 1518-1520.
} 
activated by heating at $300{ }^{\circ} \mathrm{C}$ for 24 hours. Indicated molarities of $n$-BuLi were determined by titration with diphenyl acetic acid and 2,2'dipyridyl. TMSOTf was prepared according to the procedure described by Demuth and was stored over poly-vinyl pyridine. ${ }^{2}$ 2,6-Lutidine and $N, N$ dimethylaniline were distilled from $\mathrm{CaH}_{2}$ under reduced pressure. IBX was prepared according to the procedure described by Santagostino. ${ }^{3}$ Liquid chromatography was performed using forced flow (flash chromatography) of the indicated solvent system with Fisher, Bodman or Sorbent Technologies reagent silica gel 60 (230-400 mesh). ${ }^{4}$ Thin layer chromatography (TLC) was performed on Whatman $250 \mu \mathrm{m}$ layer $6 \AA$ glass-backed silica gel plates. Eluted plates were visualized using cerric ammonium molybdate, $p$-anisaldehyde or potassium permanganate TLC stains. Medium Pressure Liquid Chromatography (MPLC) was performed on an Isco CYGNET or a Biotage Flash40i instrument.

Instrumentation. Optical rotations were measured using a JASCO DIP-370 digital polarimeter. Infrared spectra were recorded on a MIDAC Grams/Prospect FTIR spectrometer. Chiral HPLC analysis was performed on a Hewlett Packard Model 1050 Series instrument with a $0.46 \mathrm{~cm}$ x 25 cm CHIRALCEL OD-H or a $0.46 \mathrm{~cm}$ x $25 \mathrm{~cm}$ CHIRALCEL AS column. ${ }^{1} \mathrm{H}$ NMR spectra were recorded at 400 or $500 \mathrm{MHz}$ on Bruker NMR spectrometers and were referenced to either tetramethylsilane or residual protio solvent peaks. ${ }^{13} \mathrm{C}$ NMR spectra were recorded at 100 or 125 MHz on Bruker NMR spectrometers. Coupling constants refer to apparent multiplicities, indicated as follows: $\mathrm{s}$ (singlet); $\mathrm{d}$ (doublet); $\mathrm{t}$ (triplet); q (quartet); $\mathrm{m}$ (multiplet); dd (doublet of doublets); etc. Mass spectra were measured on a MicroMass Analytical 7070E, a MicroMass

\footnotetext{
${ }^{2}$ Demuth, M.; Mikhail, G. Synthesis 1982, 827.

${ }^{3}$ Frigerio, M.; Santagostino, M.; Sputore, S. J. Org. Chem. 1999, 64, 4537-4538.

${ }^{4}$ Still, W. C.; Kahn, M.; Mitra, M. J. Org. Chem. 1978, 43, 2923-2925.
} 
AutoSpec E or a MicroMass LCT Electrospray spectrometer. Combustion analyses were performed by M-H-W Laboratories (Phoenix, AZ).

First Route to Product 23.

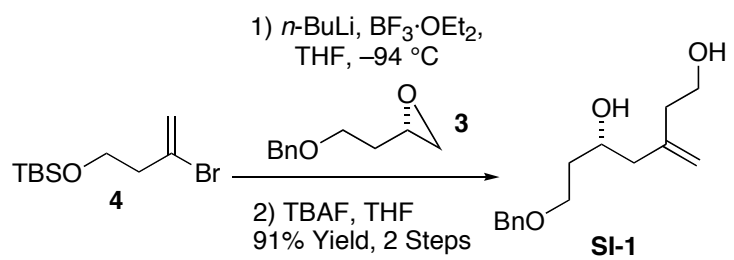

Diol SI-1. To a cooled $\left(-94^{\circ} \mathrm{C}\right)$ solution of vinyl bromide $4^{5}$ (19.9 g, 75.4 mmol, 1.5 equiv) in $200 \mathrm{~mL}$ of tetrahydrofuran was added $n$-BuLi $(29.6 \mathrm{~mL}, 2.55 \mathrm{M}$ in hexanes, $75.4 \mathrm{mmol}, 1.5$ equiv) dropwise via syringe. The reaction mixture was stirred for 10 minutes and then epoxide $\mathbf{3}^{6}$ (8.9 g, $49.9 \mathrm{mmol}, 1.0$ equiv) was added in $30 \mathrm{~mL}$ of tetrahydrofuran. After stirring for an additional 10 minutes, $\mathrm{BF}_{3} \cdot \mathrm{OEt}_{2}(7.0 \mathrm{~mL}, 54.9 \mathrm{mmol}, 1.1$ equiv) was added dropwise via syringe. The reaction mixture was maintained at $-94{ }^{\circ} \mathrm{C}$ for another 15 minutes and was then warmed to $78{ }^{\circ} \mathrm{C}$. The reaction mixture was gradually warmed to $-40{ }^{\circ} \mathrm{C}$ over the course of 15 hours whereupon saturated aqueous $\mathrm{NaHCO}_{3}(50 \mathrm{~mL})$ and $\mathrm{H}_{2} \mathrm{O}(500 \mathrm{~mL})$ were added. After warming to $25{ }^{\circ} \mathrm{C}$, the aqueous phase was extracted with ether $(4 \times 150 \mathrm{~mL})$, and the combined organic extracts were washed with brine $\left(3 \times 50 \mathrm{~mL}\right.$ ), dried over anhydrous $\mathrm{MgSO}_{4}$, filtered and concentrated in vacuo. The resulting material $(18.2 \mathrm{~g}, 49.9 \mathrm{mmol})$ was dissolved in $40 \mathrm{~mL}$ of tetrahydrofuran, and TBAF (59.9 mL, 1.0 M in THF, 59.9 mmol, 1.2 equiv) was added dropwise via syringe. The reaction mixture was stirred for 30 minutes, diluted with ethyl acetate $(10 \mathrm{~mL})$ and concentrated in vacuo. The resulting crude oil was purified by flash chromatography on silica gel (50\% ether/hexanes to $100 \%$ ether and then $50 \%$ ethyl acetate/hexanes to $100 \%$ ethyl

${ }^{5}$ Basil, L.F.; Nakano, H.; Frutos, R.; Kopach, M.; Meyers, A.I. Synthesis 2002, 14, 2064-2074. ${ }^{6}$ Schaus, S.E.; Brandes, B.D.; Larrow, J.F.; Tokunaga, M.; Hansen, K.B.; Gould, A.E.; Furrow, M.E.; Jacobsen, E.N. J. Am. Chem. Soc. 2002, 124, 1307-1315. 
acetate) to afford diol SI-1 (11.40 g, 91\%, 2 steps) as a colorless oil: $[\alpha]{ }_{\mathrm{D}}^{24}+14.3(\mathrm{c} 1.12$, $\mathrm{CHCl}_{3}$ ); IR (neat) 3390, 2936, 2867, 1644, 1077; ${ }^{1} \mathrm{H}$ NMR (400 MHz, $\left.\mathrm{CDCl}_{3}\right) \delta$ 7.40-7.27 (m, $5 \mathrm{H}), 4.98(\mathrm{~s}, 2 \mathrm{H}), 4.53(\mathrm{~s}, 2 \mathrm{H}), 4.05-3.98(\mathrm{~m}, 1 \mathrm{H}), 3.78-3.71(\mathrm{~m}, 3 \mathrm{H}), 3.69-3.61(\mathrm{~m}, 1 \mathrm{H})$, 2.49 (br s, $2 \mathrm{H}), 2.40-2.32$ (m, $2 \mathrm{H}), 2.27-2.19$ (m, $2 \mathrm{H}), 1.86-1.74(\mathrm{~m}, 2 \mathrm{H}) ;{ }^{13} \mathrm{C}$ NMR (125 $\left.\mathrm{MHz}, \mathrm{CDCl}_{3}\right) \delta 143.5,137.8,128.4,127.7,127.6,114.4,73.2,69.3,68.7,60.5,43.8,39.1,36.3$; HRMS (FAB) $m / z$ calcd for $\mathrm{C}_{15} \mathrm{H}_{22} \mathrm{O}_{3}[\mathrm{M}+\mathrm{H}]^{+}$251.1647, found 251.1653. Anal. Calcd for $\mathrm{C}_{15} \mathrm{H}_{22} \mathrm{O}_{3}$ : C, 71.97; H, 8.86;. Found: C, 77.02; H, 8.77.

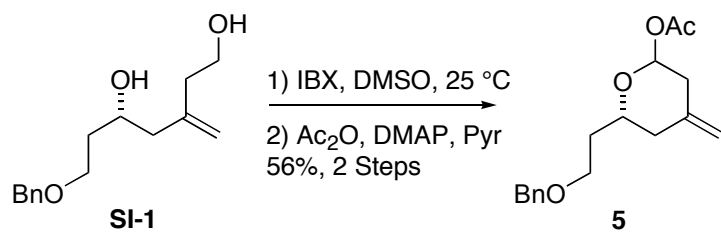

Acetoxy Ether 5. This procedure was adapted from work reported by Corey. ${ }^{7}$ To a suspension of IBX (2.69 g, $9.59 \mathrm{mmol}, 1.2$ equiv) in $15 \mathrm{~mL}$ of acetone and $8 \mathrm{~mL}$ of DMSO was added diol SI-1 (2.00 g, $7.99 \mathrm{mmol}, 1.0$ equiv) in $7 \mathrm{~mL}$ of acetone ( $4 \mathrm{~mL}, 3 \mathrm{~mL}$ rinse) dropwise via cannula. After stirring for 3.5 hours at $25{ }^{\circ} \mathrm{C}$, the reaction mixture was diluted with $\mathrm{H}_{2} \mathrm{O}(80 \mathrm{~mL})$ and ethyl acetate $(80 \mathrm{~mL})$. Following filtration of the layers through a pad of celite, the aqueous phase was extracted with ethyl acetate $(3 \times 50 \mathrm{~mL})$; and the combined organic extracts were washed with brine $(100 \mathrm{~mL})$, dried over anhydrous $\mathrm{MgSO}_{4}$, filtered and concentrated in vacuo. The resulting crude oil was purified by flash chromatography on silica gel deactivated with $2 \%$ triethylamine (35\% ethyl acetate/hexanes to $50 \%$ ethyl acetate/hexanes) to afford the lactol (1.70 g) as an orange oil. This material was then dissolved in dichloromethane $(20 \mathrm{~mL})$, and after cooling to $0{ }^{\circ} \mathrm{C}$, pyridine $(2.20 \mathrm{~mL}, 27.2 \mathrm{mmol}, 4.0$ equiv), acetic anhydride $(1.42 \mathrm{~mL}, 15.1$

${ }^{7}$ For the primary selective oxidation of 1,4- and 1,5-diols with IBX, see: (a) Corey, E.J.; Palani, A. Tetrahedron Lett. 1995, 38, 3485-3488.(b) Corey, E.J.; Palani, A. Tetrahedron Lett. 1995, 36, 7945-7948. 
mmol, 2.2 equiv) and DMAP $(1.1 \mathrm{~g}, 8.80 \mathrm{mmol}, 1.3$ equiv) were added. The reaction mixture was stirred for 2.5 hours at $0{ }^{\circ} \mathrm{C}$ and was then diluted with $1 \mathrm{~N}$ saturated aqueous $\mathrm{NaHSO}_{4}(25$ $\mathrm{mL})$. Following extraction of the aqueous phase with ethyl acetate $(3 \times 50 \mathrm{~mL})$, the combined organic extracts were washed with saturated aqueous $\mathrm{NaHCO}_{3}(80 \mathrm{~mL})$, brine $(80 \mathrm{~mL})$, dried over anhydrous $\mathrm{MgSO}_{4}$, filtered and concentrated in vacuo. The resulting crude oil was purified by flash chromatography on silica gel deactivated with $2 \%$ triethylamine (10\% ethyl acetate/hexanes to $20 \%$ ethyl acetate/hexanes) to afford acetoxy ether $\mathbf{5}(1.3 \mathrm{~g}, 56 \% 2$ steps $)$ as a colorless oil. The reported data represents the 1.5:1 mixture of diastereomers: IR (neat) 3075, 3031, 2949, 1749, $1657 \mathrm{~cm}^{-1} ;{ }^{1} \mathrm{H}$ NMR $\left(500 \mathrm{MHz}, \mathrm{CDCl}_{3}\right) \delta$ 7.36-7.27 (m, $\left.5 \mathrm{H}\right), 6.19(\mathrm{~d}, J=3.6$ $\mathrm{Hz}, 0.4 \mathrm{H}), 5.59(\mathrm{dd}, J=9.6,2.7 \mathrm{~Hz}, 0.6 \mathrm{H}), 4.83(\mathrm{~d}, J=1.7 \mathrm{~Hz}, 1.6 \mathrm{H}), 4.80(\mathrm{~d}, J=1.8 \mathrm{~Hz}, 0.4$ $\mathrm{H}), 4.53-4.47(\mathrm{~m}, 2 \mathrm{H}), 4.05-4.00(\mathrm{~m}, 0.4 \mathrm{H}), 3.69-3.63(\mathrm{~m}, 0.6 \mathrm{H}), 3.62-3.54(\mathrm{~m}, 2 \mathrm{H}), 2.50-$ $2.46(\mathrm{~m}, 1 \mathrm{H}), 2.37-2.30(\mathrm{~m}, 1 \mathrm{H}), 2.27-2.21(\mathrm{~m}, 1 \mathrm{H}), 2.11(\mathrm{~s}, 1.8 \mathrm{H}), 2.09-2.04(\mathrm{~m}, 0.4 \mathrm{H})$, 2.03 (s, 1.2 H), 1.99-1.97 (m, 0.4 H), 1.92 (dddd, $J=13.9,8.1,5.5,5.5 \mathrm{~Hz}, 0.6 \mathrm{H}), 1.87-1.79$ (m, 1.6 H); $\left.{ }^{13} \mathrm{C} \mathrm{NMR} \mathrm{(125} \mathrm{MHz,} \mathrm{CDCl}_{3}\right) \delta$ 169.4, 168.7, 140.5, 139.0, 138.2(2), 128.1(2), 127.4, 127.3(2), 111.6, 111.0, 94.3, 92.4, 73.5, 73.0(2), 69.2, 66.4, 66.3, 39.8, 39.6, 39.5, 37.7, 35.9, 21.4, 21.3; HRMS (CI/ammonia) $\mathrm{m} / z$ calcd for $\mathrm{C}_{17} \mathrm{H}_{22} \mathrm{O}_{4}[\mathrm{M}-\mathrm{OAc}]^{+} 231.1385$, found 231.1381. Anal. Calcd for $\mathrm{C}_{17} \mathrm{H}_{22} \mathrm{O}_{4}: \mathrm{C}, 70.32 ; \mathrm{H}, 7.64$. Found: $\mathrm{C}, 70.58 ; \mathrm{H}, 7.44$.

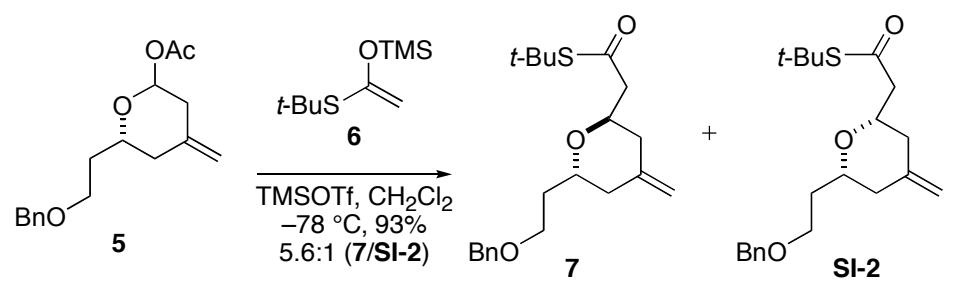


Thioesters 7 and SI-2. To a cooled $\left(-78^{\circ} \mathrm{C}\right)$ solution of acetoxy acetal 5 (3.18 g, $11.0 \mathrm{mmol}$, 1.0 equiv) and thioketene acetal 6 (2.69 g, $13.1 \mathrm{mmol}, 1.2$ equiv) in $50 \mathrm{~mL}$ of dichloromethane was added TMSOTf (198 $\mu \mathrm{L}, 1.09 \mathrm{mmol}, 0.099$ equiv) dropwise via syringe. After stirring for 2 hours at $-78{ }^{\circ} \mathrm{C}$, the reaction mixture was diluted with a $2: 1$ mixture of triethylamine and methanol $(1.5 \mathrm{~mL})$. The reaction mixture was then warmed to $25^{\circ} \mathrm{C}$ and saturated aqueous $\mathrm{NaHCO}_{3}(50 \mathrm{~mL})$ was added. Following extraction of the aqueous phase with dichloromethane ( $2 \times 50 \mathrm{~mL}$ ), the combined organic extracts were dried over anhydrous $\mathrm{Na}_{2} \mathrm{SO}_{4}$, filtered and concentrated in vacuo. The resulting crude oil was purified by medium pressure liquid chromatography (MPLC) on silica gel (5\% ether/hexanes to $30 \%$ ether/hexanes) to afford antithioester 7 (3.15 g, 79\%) and syn-thioester SI-2 both as a colorless oils.

Data for 7: $[\alpha]{ }_{\mathrm{D}}^{24}-61.7$ (c 1.26, $\mathrm{CHCl}_{3}$ ); IR (neat/NaCl) 3071, 2959, 2863, 1681, $1454 \mathrm{~cm}^{-1} ;{ }^{1} \mathrm{H}$ NMR $\left(500 \mathrm{MHz}, \mathrm{CDCl}_{3}\right) \delta 7.37-7.24(\mathrm{~m}, 5 \mathrm{H}), 4.79$ (br s, $\left.2 \mathrm{H}\right), 4.50(\mathrm{ABq}, J=11.9 \mathrm{~Hz}, \Delta v=$ $9.4 \mathrm{~Hz}, 2 \mathrm{H}), 4.31-4.26$ (m, $1 \mathrm{H}), 3.99-3.94$ (m, $1 \mathrm{H}), 3.56-3.54$ (m, $2 \mathrm{H}), 2.74$ (dd, J = 14.5, 7.9 $\mathrm{Hz}, 1 \mathrm{H}), 2.55(\mathrm{dd}, J=14.5,5.8 \mathrm{~Hz}, 1 \mathrm{H}), 2.37(\mathrm{dd}, J=12.5,4.5 \mathrm{~Hz}, 1 \mathrm{H}), 2.35(\mathrm{dd} J=12.6 \mathrm{~Hz}$, $1 \mathrm{H}), 2.05-1.97$ (m, $2 \mathrm{H}), 1.94$ (dddd, $J=14.9,9.2,5.8,5.8 \mathrm{~Hz}, 1 \mathrm{H}), 1.67$ (dddd, $J=14.4,7.3$, 7.3, $4.4 \mathrm{~Hz}, 1 \mathrm{H}), 1.46$ (s, $9 \mathrm{H}) ;{ }^{13} \mathrm{C} \mathrm{NMR}\left(125 \mathrm{MHz}, \mathrm{CDCl}_{3}\right) \delta 197.1,140.8,138.2,128.0,127.3$, 127.2, 110.8, 73.0, 69.6, 69.3, 66.9, 48.2, 48.1, 39.7, 39.2, 33.7, 29.8; HRMS (CI/ammonia) $\mathrm{m} / \mathrm{z}$ calcd for $\mathrm{C}_{21} \mathrm{H}_{30} \mathrm{O}_{3} \mathrm{~S}[\mathrm{M}+\mathrm{H}]^{+}$363.1994, found 363.2002. Anal. Calcd for $\mathrm{C}_{21} \mathrm{H}_{30} \mathrm{O}_{3} \mathrm{~S}$ : C, 69.58; H, 8.34. Found: C, 69.70; H, 8.19.

Data for SI-2: $\left[\alpha{ }^{24}-23.4\left(c 1.26, \mathrm{CHCl}_{3}\right)\right.$; IR (neat/NaCl) 3071, 3030, 2922, 2863, 1682, 1454 $\mathrm{cm}^{-1} ;{ }^{1} \mathrm{H}$ NMR $\left(400 \mathrm{MHz}, \mathrm{CDCl}_{3}\right) \delta$ 7.37-7.25 (m, $\left.5 \mathrm{H}\right), 4.73(\mathrm{~d}, J=1.6 \mathrm{~Hz}, 1 \mathrm{H}), 4.72(\mathrm{~d}, J=$ 
$1.6 \mathrm{~Hz}, 1 \mathrm{H}), 4.48$ (s, $2 \mathrm{H}), 3.73$ (dddd, $J=11.2,7.7,5.5,2.3 \mathrm{~Hz}, 1 \mathrm{H}), 3.60-3.57$ (m, $2 \mathrm{H}), 3.48-$ $3.43(\mathrm{~m}, 1 \mathrm{H}), 2.72(\mathrm{dd}, J=14.5,7.8 \mathrm{~Hz}, 1 \mathrm{H}), 2.52(\mathrm{dd}, J=14.5,5.4 \mathrm{~Hz}, 1 \mathrm{H}), 2.26-2.17(\mathrm{~m}, 2$ H), $1.94($ app t, $J=12.5 \mathrm{~Hz}, 2 \mathrm{H}), 1.86-1.72(\mathrm{~m}, 2 \mathrm{H}), 1.46(\mathrm{~s}, 9 \mathrm{H}) ;{ }^{13} \mathrm{C}$ NMR $(125 \mathrm{MHz}$, $\left.\mathrm{CDCl}_{3}\right) \delta 197.0,143.3,138.3,128.1,127.4,127.2,109.1,75.4,74.9,73.0,66.8,50.9,48.1,40.7$, 40.3, 36.5, 29.9; HRMS (CI/ammonia) $\mathrm{m} / z$ calcd for $\mathrm{C}_{21} \mathrm{H}_{30} \mathrm{O}_{3} \mathrm{~S}[\mathrm{M}+\mathrm{H}]^{+}$363.1994, found 363.1996. Anal. Calcd for $\mathrm{C}_{21} \mathrm{H}_{30} \mathrm{O}_{3} \mathrm{~S}: \mathrm{C}, 69.58 ; \mathrm{H}$, 8.34. Found: C, 69.76; H, 8.17.

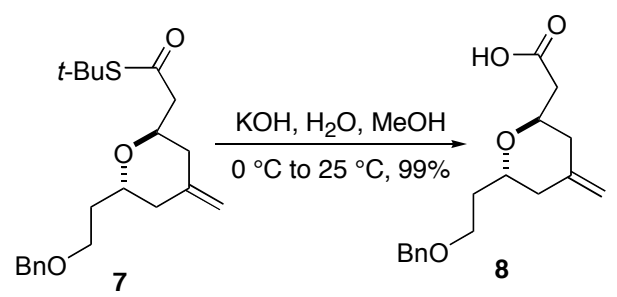

Carboxylic Acid 8. To a solution of tert-butyl thioester 7 (1.80 g, $4.96 \mathrm{mmol}, 1.0$ equiv) in $\mathrm{MeOH}(18 \mathrm{~mL})$ at $0{ }^{\circ} \mathrm{C}$ was added $50 \%$ aqueous $\mathrm{KOH}(2 \mathrm{~mL})$. The reaction mixture was warmed to $25{ }^{\circ} \mathrm{C}$ and stirred for 45 minutes whereupon $\mathrm{H}_{2} \mathrm{O}(40 \mathrm{~mL})$ was added. The aqueous phase was extracted with dichloromethane ( $3 \times 40 \mathrm{~mL})$, and then the $\mathrm{pH}$ was adjusted to 1 with $1 \mathrm{~N}$ aqueous $\mathrm{HCl}(50 \mathrm{~mL})$. The aqueous phase was then extracted with ethyl acetate $(3 \times 50 \mathrm{~mL})$, the combined organic extracts were dried over anhydrous $\mathrm{MgSO}_{4}$, filtered and concentrated in vacuo to afford carboxylic acid $\mathbf{8}(1.43 \mathrm{~g}, 99 \%)$ as a colorless oil: $[\alpha]{ }_{\mathrm{D}}^{24}-58.2\left(\mathrm{c} 1.02, \mathrm{CH}_{2} \mathrm{Cl}_{2}\right)$; IR (neat) 3070, 3031, 2944, 2685, 1732, $1711 \mathrm{~cm}^{-1} ;{ }^{1} \mathrm{H}$ NMR (400 MHz, $\left.\mathrm{CDCl}_{3}\right)$ 8 7.35-7.22 (m, $5 \mathrm{H}), 4.81(\mathrm{br} \mathrm{s}, 2 \mathrm{H}), 4.49(\mathrm{ABq}, J=12.0 \mathrm{~Hz}, \Delta v=8.0 \mathrm{~Hz}, 2 \mathrm{H}), 4.27-4.21(\mathrm{~m}, 1 \mathrm{H}), 4.09-4.03$ (m, $1 \mathrm{H}), 3.56-3.51(\mathrm{~m}, 2 \mathrm{H}), 2.64(\mathrm{dd}, J=15.5,8.4 \mathrm{~Hz}, 1 \mathrm{H}), 2.48(\mathrm{dd}, J=15.5,5.2 \mathrm{~Hz}, 1 \mathrm{H})$, $2.41(\mathrm{dd}, J=8.6,4.4 \mathrm{~Hz}, 1 \mathrm{H}), 2.38(\mathrm{dd}, J=8.4,4.4 \mathrm{~Hz}, 1 \mathrm{H}), 2.06(\mathrm{dd}, J=13.6,6.4 \mathrm{~Hz}, 1 \mathrm{H})$, $2.03(\mathrm{dd}, J=13.2,6.4 \mathrm{~Hz}, 1 \mathrm{H}), 1.95$ (dddd, $J=14.5,9.1,5.5,5.5 \mathrm{~Hz}, 1 \mathrm{H}), 1.69$ (dddd, $J=14.3$, 
6.8, 6.8, 4.6 Hz, $1 \mathrm{H}) ;{ }^{13} \mathrm{C} \mathrm{NMR}\left(125 \mathrm{MHz}, \mathrm{CDCl}_{3}\right) \delta 176.4,140.5,138.0,128.1,127.4,127.3$, 111.0, 72.9, 69.8, 68.6, 66.6, 39.5, 39.2, 38.7, 33.3; HRMS (CI/ammonia) $\mathrm{m} / z$ calcd for $\mathrm{C}_{17} \mathrm{H}_{22} \mathrm{O}_{4}[\mathrm{M}+\mathrm{H}]^{+}$291.1596, found 291.1602. Anal. Calcd for $\mathrm{C}_{17} \mathrm{H}_{22} \mathrm{O}_{4}: \mathrm{C}, 70.32 ; \mathrm{H}, 7.64$. Found: C, 70.09; H, 7.68.

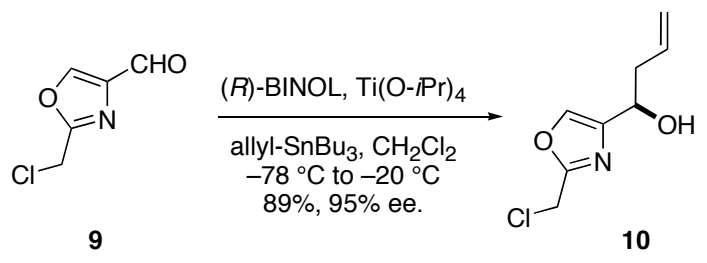

Homoallylic Alcohol 10. The general procedure for the asymmetric allylation of aldehydes developed by Keck was followed. ${ }^{8}$ To a solution of $(R)$-BINOL (214 mg, 0.749 mmol, 0.1 equiv) in dichloromethane $(12 \mathrm{~mL})$ at $25{ }^{\circ} \mathrm{C}$ was added powdered $4 \AA$ Linde molecular sieves $(2.00 \mathrm{~g})$ and $\operatorname{Ti}(\mathrm{O}-i \mathrm{Pr})_{4}(223 \mu \mathrm{L}, 0.749 \mathrm{mmol}, 0.1$ equiv). The resulting dark red suspension was then gently refluxed at $43{ }^{\circ} \mathrm{C}$ for 1 hour. After cooling the catalyst suspension to $25^{\circ} \mathrm{C}$, aldehyde $9^{9}$ (1.09 g, $7.49 \mathrm{mmol}, 1.0$ equiv) was added as a solution in dichloromethane (6 mL) via cannula. The reaction mixture was then cooled to $-78{ }^{\circ} \mathrm{C}$, and allyltributyltin $(2.98 \mathrm{~g}, 8.99 \mathrm{mmol}, 1.2$ equiv) was added. The reaction mixture was then placed in a $-20{ }^{\circ} \mathrm{C}$ freezer for 66 hours without stirring whereupon aqueous $\mathrm{NaHCO}_{3}(20 \mathrm{~mL})$ was added. After stirring for 1 hour at 25 ${ }^{\circ} \mathrm{C}$, the reaction mixture was filtered through a celite cake and extracted with dichloromethane (3 x $40 \mathrm{~mL}$ ). The combined organic extracts were dried over anhydrous $\mathrm{MgSO}_{4}$, filtered and concentrated in vacuo. The resulting dark red oil was purified by flash chromatography on silica gel (35\% ethyl acetate/hexanes) to afford $\mathbf{1 0}(1.25 \mathrm{~g}, 89 \%)$ as a colorless oil: $[\alpha]{ }_{\mathrm{D}}^{24}+35.7(c$ 1.17, $\mathrm{CHCl}_{3}$ ); IR (neat) 3366, 3079, 3027, 2979, 2911, 1642, 1570, 1339, 1266, 1099, 1067; ${ }^{1} \mathrm{H}$ $\operatorname{NMR}\left(400 \mathrm{MHz}, \mathrm{CDCl}_{3}\right) \delta 7.57(\mathrm{~s}, 1 \mathrm{H}), 5.81$ (dddd, $\left.J=17.2,10.4,6.8,6.8 \mathrm{~Hz}, 1 \mathrm{H}\right), 5.22-5.16$

\footnotetext{
${ }^{8}$ Keck, G.E.; Tarbet, K.H.; Geraci, L.S. J. Am. Chem. Soc. 1993, 115, 8467-8468.

${ }^{9}$ White, J.D.; Kranemann, C.L.; Kuntyong, P. Org. Lett. 2001, 3, 4003-4006.
} 
(m, 2H), $4.73(\mathrm{dd}, J=7.6,5.2 \mathrm{~Hz}, 1 \mathrm{H}), 4.58(\mathrm{~s}, 2 \mathrm{H}), 2.68-2.61(\mathrm{~m}, 1 \mathrm{H}), 2.54(\mathrm{ddd}, J=14.8$,

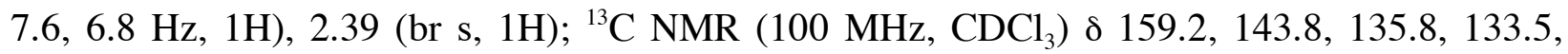
118.8, 66.2, 40.6, 35.7; HRMS (CI/isobutane) $\mathrm{m} / \mathrm{z}$ calcd for $\mathrm{C}_{8} \mathrm{H}_{10} \mathrm{ClNO}_{2}[\mathrm{M}]^{+}$187.0400, found 187.0401. Anal. Calcd for $\mathrm{C}_{8} \mathrm{H}_{10} \mathrm{ClNO}_{2}$ : C, 51.21; H, 5.37; N, 7.47. Found: C, 51.10; H, 5.32; $\mathrm{N}, 7.41$. The optical purity of $\mathbf{1 0}$ was established to be $>95 \%$ ee by chiral HPLC analysis (Chiralcel OD-H; 90:10 hexane/isopropanol; $1.00 \mathrm{~mL} / \mathrm{min} . ; \mathrm{t}_{\mathrm{R}}(R$-isomer $)=8.89$ min.; $\mathrm{t}_{\mathrm{R}}(S$ isomer $)=13.32 \mathrm{~min}$. .

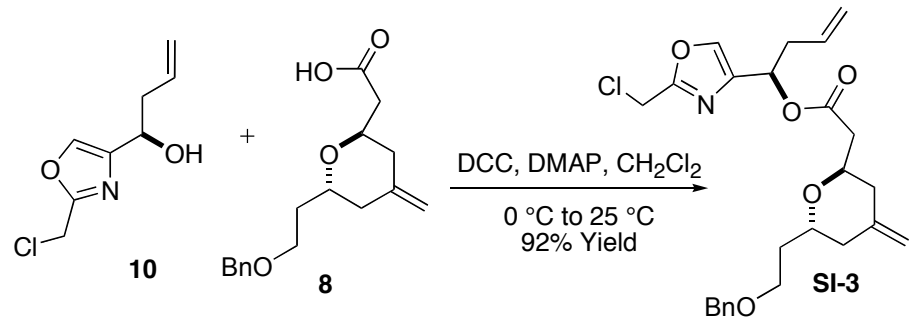

Ester SI-3. To a cooled $\left(0^{\circ} \mathrm{C}\right)$ solution of carboxylic acid 8 (950 mg, $3.27 \mathrm{mmol}, 1.0$ equiv) and homoallylic alcohol 10 (713 mg, $3.80 \mathrm{mmol}, 1.2$ equiv) in $20 \mathrm{~mL}$ of dichloromethane was added DCC (1.05 g, $5.09 \mathrm{mmol}, 1.6$ equiv) and DMAP (78.0 mg, $0.638 \mathrm{mmol}, 0.20$ equiv). The reaction mixture was then warmed to $25^{\circ} \mathrm{C}$ and stirred for 12 hours whereupon dichloromethane (20 mL) was added. The suspension was then filtered through a small plug of celite to remove the insoluble dicyclohexylurea. The organic phase was washed with $1 \mathrm{~N}$ aqueous $\mathrm{HCl}(2 \times 10$ $\mathrm{mL})$, saturated aqueous $\mathrm{NaHCO}_{3}(2 \times 20 \mathrm{~mL})$, brine $(1 \times 10 \mathrm{~mL})$, dried over anhydrous $\mathrm{MgSO}_{4}$, filtered and concentrated in vacuo. The resulting solid material was diluted with hexanes and filtered to remove remaining dicyclohexylurea. Concentration of the filtrate in vacuo provided a crude oil that was purified by flash chromatography on silica gel (10 ethyl acetate/hexanes to $50 \%$ ethyl acetate/hexanes) to afford ester SI-3 (1.35 g, 92\%) as a colorless oil: $[\alpha]{ }_{\mathrm{D}}^{24}-5.11(c$ 
1.12, $\left.\mathrm{CHCl}_{3}\right)$; IR (neat) 3074, 2944, 2863, $1738 \mathrm{~cm}^{-1} ;{ }^{1} \mathrm{H}$ NMR $\left(500 \mathrm{MHz}, \mathrm{CDCl}_{3}\right) \delta 7.60(\mathrm{~d}, J=$ $0.7 \mathrm{~Hz}, 1 \mathrm{H}), 7.35-7.25(\mathrm{~m}, 5 \mathrm{H}), 5.84(\mathrm{t}, J=6.7 \mathrm{~Hz}, 1 \mathrm{H}), 5.72(\mathrm{dddd}, J=17.1,10.2,7.0,7.0$ Hz, $1 \mathrm{H}), 5.14-5.09$ (m, $2 \mathrm{H}), 4.79$ (br s, $1 \mathrm{H}), 4.78$ (br s, $1 \mathrm{H}), 4.55$ (s, $2 \mathrm{H}), 4.46$ (ABq, $J=12.0$ $\mathrm{Hz}, \Delta v=9.9 \mathrm{~Hz}, 2 \mathrm{H}), 4.29-4.24(\mathrm{~m}, 1 \mathrm{H}), 3.97-3.94(\mathrm{~m}, 1 \mathrm{H}), 3.49(\mathrm{dd}, J=7.0,6.0 \mathrm{~Hz}, 2 \mathrm{H})$, 2.76-2.67 (m, $2 \mathrm{H}), 2.61(\mathrm{dd}, J=14.9,7.9 \mathrm{~Hz}, 1 \mathrm{H}), 2.48(\mathrm{dd}, J=14.9,6.0 \mathrm{~Hz}, 1 \mathrm{H}), 2.39$ (dd, $J$ $=13.3,4.4 \mathrm{~Hz}, 1 \mathrm{H}), 2.35(\mathrm{dd}, J=13.3,4.3 \mathrm{~Hz}, 1 \mathrm{H}), 2.04(\mathrm{dd}, J=13.3,6.2 \mathrm{~Hz}, 1 \mathrm{H}), 2.00(\mathrm{dd}, J$ $=12.9,6.3 \mathrm{~Hz}, 1 \mathrm{H}), 1.90$ (dddd, $J=14.4,8.6,5.9,5.9 \mathrm{~Hz}, 1 \mathrm{H}$ ), 1.67 (dddd, $J$ = 14.2, 7.1, 7.1, $4.9 \mathrm{~Hz}, 1 \mathrm{H}) ;{ }^{13} \mathrm{C}$ NMR $\left(125 \mathrm{MHz}, \mathrm{CDCl}_{3}\right) \delta 170.3,159.2,141.0,139.8,138.5,137.5,132.6$, $128.3,127.6,127.4,118.5,111.0,72.9,69.6,68.9,67.6,66.7,39.4,39.1,38.8,37.4,35.6,33.4$ HRMS (FAB) $m / z$ calcd for $\mathrm{C}_{25} \mathrm{H}_{30} \mathrm{ClNO}_{5}[\mathrm{M}+\mathrm{H}]^{+}$460.1892, found 460.1892. Anal. Calcd for $\mathrm{C}_{25} \mathrm{H}_{30} \mathrm{ClNO}_{5}: \mathrm{C}, 65.28 ; \mathrm{H}, 6.57 ; \mathrm{N}, 3.05$. Found: $\mathrm{C}, 65.41 ; \mathrm{H}, 6.41 ; \mathrm{N}, 3.14$.

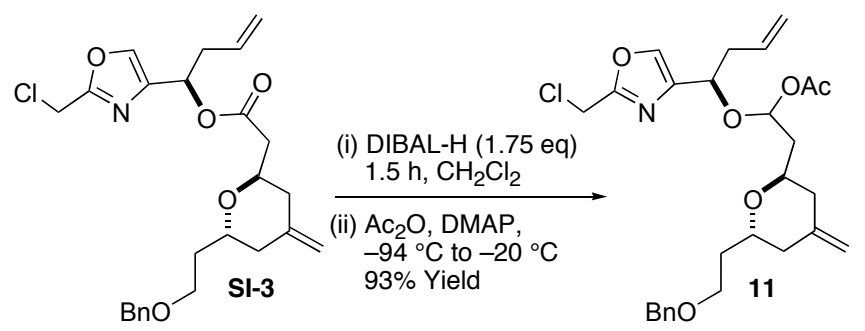

Acetoxy Ether 11. Ester SI-3 (1.00 g, $2.17 \mathrm{mmol}, 1.0$ equiv) was dissolved in $15 \mathrm{~mL}$ of dichloromethane and cooled to $-94{ }^{\circ} \mathrm{C}$ (hexanes/liquid $\mathrm{N}_{2}$ slush bath). To this cooled solution was added DIBAL-H (3.80 mL, 1.0 $\mathrm{M}$ in hexanes, $3.80 \mathrm{mmol}$, 1.75 equiv) dropwise via syringe. The reaction mixture was stirred for 1.5 hours at $-94{ }^{\circ} \mathrm{C}$ and then acetic anhydride $(1.23 \mathrm{~mL}$, $13.0 \mathrm{mmol}, 6.0$ equiv), DMAP (530 $\mathrm{mg}, 4.35 \mathrm{mmol}, 2.0$ equiv) as a solution in dichloromethane $(5 \mathrm{~mL})$ and pyridine $(527 \mu \mathrm{L}, 6.51 \mathrm{mmol}, 3.0$ equiv) were added. The reaction mixture was stirred at $-94{ }^{\circ} \mathrm{C}$ for 10 minutes and was then warmed to $-78{ }^{\circ} \mathrm{C}$ and maintained at this 
temperature for 10 hours. The reaction mixture was allowed to warm to $-20{ }^{\circ} \mathrm{C}$ over the course of 5 hours. After stirring for 0.5 hours at $-20{ }^{\circ} \mathrm{C}$, saturated aqueous $\mathrm{NH}_{4} \mathrm{Cl}(4 \mathrm{~mL})$ and saturated aqueous $\mathrm{Na} / \mathrm{K}$ tartrate $(4 \mathrm{~mL})$ were added. The resulting suspension was stirred at $-20{ }^{\circ} \mathrm{C}$ for twenty minutes, and was then diluted with ethyl acetate $(60 \mathrm{~mL})$. The organic layer was washed with ice cold $1 \mathrm{~N}$ aqueous $\mathrm{NaHSO}_{4}(60 \mathrm{~mL})$, saturated aqueous $\mathrm{NaHCO}_{3}(60 \mathrm{~mL})$, brine $(60 \mathrm{~mL})$, dried over anhydrous $\mathrm{MgSO}_{4}$, filtered and concentrated in vacuo. The resulting crude oil was purified by flash chromatography on silica gel deactivated with $2 \%$ triethylamine (10\% ether/hexanes to $50 \%$ ether/hexanes) to afford $\alpha$-acetoxy ether $\mathbf{1 1}(1.03 \mathrm{~g}, 94 \%)$ as a colorless oil. The reported data represents a 1.5:1 mixture of diastereomers: IR (neat) 3030, 2941, 2863, 1736, $1102 \mathrm{~cm}^{-1} ;{ }^{1} \mathrm{H}$ NMR (400 MHz, $\left.\mathrm{CDCl}_{3}\right) \delta 7.56(\mathrm{~s}, 0.12 \mathrm{H}), 7.49(\mathrm{~d}, J=0.8 \mathrm{~Hz}, 0.88 \mathrm{H})$, 7.35-7.23 (m, $5 \mathrm{H}), 6.06(\mathrm{dd}, J=8.6,3.0 \mathrm{~Hz}, 0.88 \mathrm{H}), 5.89(\mathrm{dd}, J=7.6,4.4 \mathrm{~Hz}, 0.12 \mathrm{H}),$, (dddd, $J=17.2,10.0,7.2,7.2 \mathrm{~Hz}, 1 \mathrm{H}), 5.11-5.04(\mathrm{~m}, 2 \mathrm{H}), 4.77$ (br s, $1 \mathrm{H}), 4.74$ (br s, $1 \mathrm{H}$ ), $4.63(\mathrm{t}, J=6.2 \mathrm{~Hz}, 1 \mathrm{H}), 4.58(\mathrm{~s}, 2 \mathrm{H}), 4.55-4.46(\mathrm{~m}, 2 \mathrm{H}), 4.15-4.09(\mathrm{~m}, 0.88 \mathrm{H}), 3.95-3.86(\mathrm{~m}$, $1 \mathrm{H}), 3.77-3.73(\mathrm{~m}, 0.12 \mathrm{H}), 3.64-3.55(\mathrm{~m}, 1.7 \mathrm{H}), 3.49-3.43(\mathrm{~m}, 0.3 \mathrm{H}), 2.69-2.53(\mathrm{~m}, 2 \mathrm{H})$, $2.42(\mathrm{dd}, J=13.2,5.2 \mathrm{~Hz}, 1 \mathrm{H}), 2.32-2.25(\mathrm{~m}, 1 \mathrm{H}), 2.11-1.88(\mathrm{~m}, 4 \mathrm{H}), 1.87(\mathrm{~s}, 3 \mathrm{H}), 1.85-1.58$ $(\mathrm{m}, 2 \mathrm{H}) ;{ }^{13} \mathrm{C}$ NMR $\left(125 \mathrm{MHz}, \mathrm{CDCl}_{3}\right) \delta 170.8,170.2,159.4,159.0,141.8,141.6,141.4,140.8$, $138.5,137.6,136.8,133.5,133.1,128.3,127.6(2), 127.4,118.2,117.5,110.5(2), 96.4,93.8$, $74.7,72.9,72.4,69.1,68.8,68.7,68.2,66.9,66.8,40.1,39.8,39.1,38.6,37.6,37.3,35.7,35.6$, 34.7, 33.9, 30.3, 21.1, 21.0; HRMS $(\mathrm{FAB}) \mathrm{m} / \mathrm{z}$ calcd for $\mathrm{C}_{27} \mathrm{H}_{34} \mathrm{ClNO}_{6}[\mathrm{M}+\mathrm{H}]^{+}$504.2152, found 504.2168. Anal. Calcd for $\mathrm{C}_{27} \mathrm{H}_{34} \mathrm{ClNO}_{6}: \mathrm{C}, 64.34 ; \mathrm{H}, 6.80 ; \mathrm{N}, 2.78$. Found: C, 64.19; H, $6.91 ; \mathrm{N}, 2.66$. 


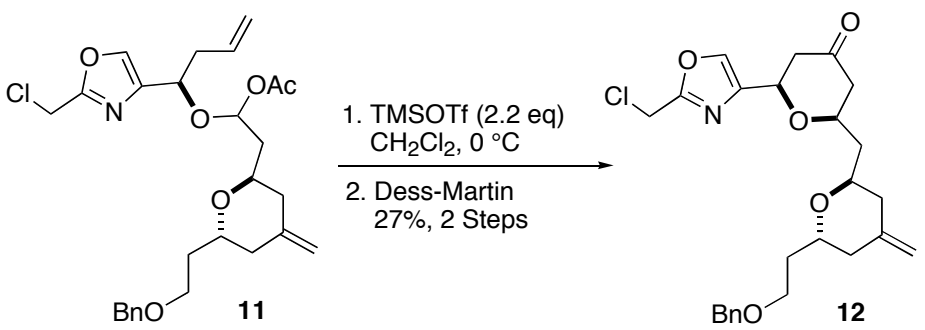

Ketone 12. To a cooled $\left(0^{\circ} \mathrm{C}\right)$ solution of acetoxy ether 11 (532 mg, $1.06 \mathrm{mmol}, 1.0$ equiv) in $10 \mathrm{~mL}$ of dichloromethane was added TMSOTf (422 $\mu \mathrm{L}, 2.33 \mathrm{mmol}, 2.2$ equiv). The reaction mixture was stirred at $0{ }^{\circ} \mathrm{C}$ for 5 minutes and was then diluted with saturated aqueous $\mathrm{NaHCO}_{3}$ (2 mL). After warming to $25^{\circ} \mathrm{C}$, the reaction mixture was diluted with additional saturated aqueous $\mathrm{NaHCO}_{3}(10 \mathrm{~mL})$ and ether $(10 \mathrm{~mL})$ and was stirred vigorously at this temperature for 12 hours. Following extraction of the aqueous phase with ether $(3 \times 20 \mathrm{~mL})$, the combined organic extracts were washed with brine $(60 \mathrm{~mL})$, dried over anhydrous $\mathrm{MgSO}_{4}$, filtered and concentrated in vacuo. The resulting crude oil was purified by flash chromatography on silica gel (50\% ether/hexanes to $75 \%$ ethyl acetate/hexanes) to provide a mixture of diastereomeric alcohols (222 mg, $0.481 \mathrm{mmol}, 45 \%$, ca. $80 \%$ purity). This material was dissolved in $4 \mathrm{~mL}$ of dichloromethane and Dess-Martin periodinane (510 mg, $1.20 \mathrm{mmol}, 2.5$ equiv) was added. The reaction mixture was stirred at $25{ }^{\circ} \mathrm{C}$ for 10 minutes and was diluted with a $1: 1$ solution of saturated aqueous $\mathrm{NaHCO}_{3}$ and $0.5 \mathrm{M} \mathrm{Na}_{2} \mathrm{~S}_{2} \mathrm{O}_{3}$. Following extraction of the aqueous phase with ethyl acetate $(3 \times 20 \mathrm{~mL})$, the combined organic extracts were dried over anhydrous $\mathrm{MgSO}_{4}$, filtered and concentrated in vacuo. The resulting crude oil was purified by flash chromatography on silica gel (20\% ethyl acetate/hexanes to $75 \%$ ethyl acetate/hexanes) to afford ketone 11 (132 mg, 27\%, 2 steps) as a colorless oil: $[\alpha]{ }_{D}^{24}-37.7$ (c 1.10, $\mathrm{CH}_{2} \mathrm{Cl}_{2}$ ); IR (neat/NaCl) 2944, 2918, 2863, 1720, $1101 \mathrm{~cm}^{-1} ;{ }^{1} \mathrm{H}$ NMR $\left(500 \mathrm{MHz}, \mathrm{CDCl}_{3}\right) \delta 7.48(\mathrm{~d}, J=0.6 \mathrm{~Hz}, 1 \mathrm{H}), 7.35-7.30$ (m, 3 H), 7.28-7.24 (m, $2 \mathrm{H}), 4.78($ br s, $1 \mathrm{H}), 4.74$ (br s, $1 \mathrm{H}), 4.57$ (s, $2 \mathrm{H}), 4.47(\mathrm{ABq}, J=12.0 \mathrm{~Hz}$, 
$\Delta v=16.3 \mathrm{~Hz}, 2 \mathrm{H}), 4.38(\mathrm{dd}, J=11.8,2.2 \mathrm{~Hz}, 1 \mathrm{H}), 4.00$ (app sextet, $J=4.9 \mathrm{~Hz}, 1 \mathrm{H}), 3.93$ (app septet, $J=4.1 \mathrm{~Hz}, 1 \mathrm{H}), 3.87-3.81(\mathrm{~m}, 1 \mathrm{H}), 3.58$ (ddd, $J=9.1,9.1,5.4 \mathrm{~Hz}, 1 \mathrm{H}), 3.49$ (ddd, $J=9.4,5.4,5.4 \mathrm{~Hz}, 1 \mathrm{H}) 2.74(\mathrm{dd}, J=14.6,11.8 \mathrm{~Hz}, 1 \mathrm{H}), 2.59-2.52(\mathrm{~m}, 2 \mathrm{H}), 2.41-2.37(\mathrm{~m}, 2$ H), $2.32(\mathrm{dd}, J=14.6,10.9 \mathrm{~Hz}, 1 \mathrm{H}), 2.26(\mathrm{ddd}, J=13.9,9.4,5.1 \mathrm{~Hz}, 1 \mathrm{H}), 2.02-1.96(\mathrm{~m}, 2 \mathrm{H})$, 1.57 (ddd, $J=13.9,7.9,4.9 \mathrm{~Hz}, 1 \mathrm{H}) ;{ }^{13} \mathrm{C} \mathrm{NMR}\left(125 \mathrm{MHz}, \mathrm{CDCl}_{3}\right) \delta 205.7,159.4,141.5,140.7$, $138.5,136.7,128.3,127.4(2), 110.8,74.3,72.8,71.3,68.4(2), 66.6,47.0,46.2,40.0,39.3,38.6$, 35.6, 34.3; HRMS (CI/Ammonia) $m / z$ calcd for $\mathrm{C}_{25} \mathrm{H}_{30} \mathrm{ClNO}_{5}[\mathrm{M}]^{+}$459.1813, found 459.1806.

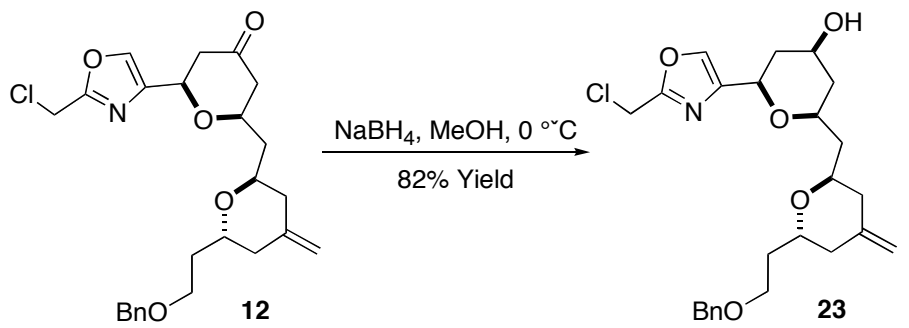

Alcohol 23. To a cooled $\left(0{ }^{\circ} \mathrm{C}\right)$ solution of ketone 12 (130 mg, $0.283 \mathrm{mmol}, 1.0$ equiv) in $3 \mathrm{~mL}$ of methanol was added $\mathrm{NaBH}_{4}(21.0 \mathrm{mg}, 0.566 \mathrm{mmol}, 2.0$ equiv $)$ as a solid in one portion. The reaction mixture was stirred at $0{ }^{\circ} \mathrm{C}$ for 10 minutes whereupon saturated aqueous $\mathrm{NH}_{4} \mathrm{Cl}(2 \mathrm{~mL})$ was added. The reaction mixture was warmed to $25^{\circ} \mathrm{C}$, diluted with ethyl acetate $(10 \mathrm{~mL})$, and additional saturated aqueous $\mathrm{NH}_{4} \mathrm{Cl}(10 \mathrm{~mL})$ was added. The reaction mixture was extracted with ethyl acetate $(2 \times 10 \mathrm{~mL})$, and the combined organic extracts were then washed with saturated aqueous $\mathrm{NaHCO}_{3}(20 \mathrm{~mL})$, brine $(20 \mathrm{~mL})$, dried over anhydrous $\mathrm{MgSO}_{4}$, filtered and concentrated in vacuo. The resulting crude oil was purified by flash chromatography on silica gel (50\% ethyl acetate/hexanes to 75\% ethyl acetate/hexanes) to afford the desired alcohol (107 mg, 82\%) as a colorless oil: $[\alpha]{ }_{\mathrm{D}}^{24}-17.8\left(\mathrm{c} 0.105, \mathrm{CH}_{2} \mathrm{Cl}_{2}\right)$; IR (neat/NaCl) 3412, 2943, 2919, 2858, 1364, $1098 \mathrm{~cm}^{-1} ;{ }^{1} \mathrm{H}$ NMR (500 MHz, $\left.\mathrm{CDCl}_{3}\right) \delta 7.50$ (s, $\left.1 \mathrm{H}\right), 7.33-7.31$ (m, $\left.3 \mathrm{H}\right), 7.28-$ 
7.24 (m, $2 \mathrm{H}), 4.76$ (br s, $1 \mathrm{H}), 4.74$ (br s, $1 \mathrm{H}), 4.56$ (s, $2 \mathrm{H}), 4.50$ (s, $2 \mathrm{H}), 4.26$ (d, J = 10.4 Hz, $1 \mathrm{H}), 4.02-3.98$ (m, $1 \mathrm{H}), 3.96-3.91$ (m, $1 \mathrm{H}), 3.83$ (dddd, $J=10.9,10.9,4.6,4.6 \mathrm{~Hz}, 1 \mathrm{H}), 3.58-$ $3.49(\mathrm{~m}, 3 \mathrm{H}), 2.38(\mathrm{dd}, J=13.2,4.7 \mathrm{~Hz}, 1 \mathrm{H}), 2.31,(\mathrm{dd}, J=13.2,3.9 \mathrm{~Hz}, 1 \mathrm{H}), 2.25-2.21(\mathrm{~m}, 1$ H), 2.14-2.07 (m, 2 H), 2.02-1.97 (m, 2 H), 1.89-1.82 (m, 2 H), 1.76-1.68 (m, $1 \mathrm{H}), 1.57-1.48$

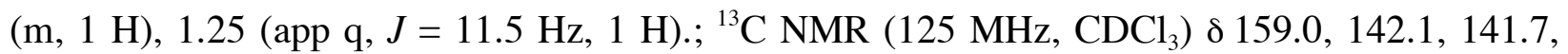
138.5, 136.2, 128.4, 127.5(2), 110.5, 73.0(2), 70.9, 68.7(2), 67.8, 66.8, 40.3, 40.0(2), 39.4, 38.6, 35.7, 34.1; HRMS (CI/Ammonia) $m / z$ calcd for $\mathrm{C}_{25} \mathrm{H}_{32} \mathrm{ClNO}_{5}[\mathrm{M}]^{+}$461.1969, found 461.1965.

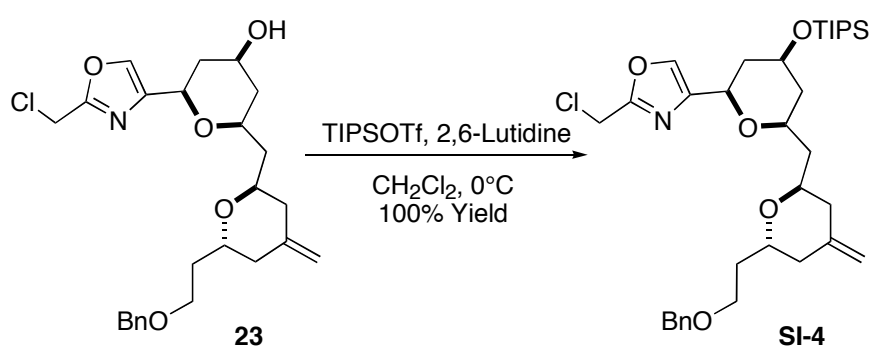

Triisopropylsilyl Ether SI-4. To a solution of alcohol 23 (100 mg, 0.216 mmol, 1.0 equiv) in 2 $\mathrm{mL}$ of dichloromethane at $25{ }^{\circ} \mathrm{C}$ was added 2,6-lutidine (126 $\mu \mathrm{L}, 1.08 \mathrm{mmol}, 5.0$ equiv) dropwise via syringe. The reaction mixture was cooled to $0{ }^{\circ} \mathrm{C}$ and TIPSOTf $(233 \mu \mathrm{L}, 0.864$ mmol, 4.0 equiv) was added dropwise via syringe. The reaction mixture was stirred at $0{ }^{\circ} \mathrm{C}$ for twenty minutes whereupon saturated aqueous $\mathrm{NaHCO}_{3}(2 \mathrm{~mL})$ was added. The reaction mixture was warmed to $25^{\circ} \mathrm{C}$ and diluted with diethyl ether $(20 \mathrm{~mL})$ and additional saturated aqueous $\mathrm{NaHCO}_{3}(20 \mathrm{~mL})$. The organic layer was then washed with $1 \mathrm{~N} \mathrm{NaHSO}_{4}(20 \mathrm{~mL})$, saturated aqueous $\mathrm{NaHCO}_{3}(20 \mathrm{~mL})$, brine $(20 \mathrm{~mL})$, dried over anhydrous $\mathrm{MgSO}_{4}$, filtered and concentrated in vacuo. The resulting crude oil was purified by flash chromatography on silica gel (10\% ether/hexanes to $50 \%$ ether/hexanes) to afford SI-4 (135 mg, 100\%) as a colorless oil: $[\alpha]{ }_{\mathrm{D}}^{24}-27.4\left(c \quad 0.475, \mathrm{CH}_{2} \mathrm{Cl}_{2}\right.$ ); IR (thin film) 2945, 2891, 2868, 1463, 1366, $1096 \mathrm{~cm}^{-1} ;{ }^{1} \mathrm{H}$ 
NMR (500 MHz, $\left.\mathrm{CDCl}_{3}\right) \delta 7.50$ (s, $\left.1 \mathrm{H}\right), 7.35-7.32$ (m, $\left.3 \mathrm{H}\right), 7.29-7.25$ (m, $\left.2 \mathrm{H}\right), 4.76$ (br s, 1 H), 4.74 (br s, $1 \mathrm{H}), 4.57$ (s, $2 \mathrm{H}), 4.50(\mathrm{~s}, 2 \mathrm{H}), 4.23$ (d, J = 10.7 Hz, $1 \mathrm{H}), 4.03-4.00$ (m, $1 \mathrm{H})$, 3.95-3.92 (m, $2 \mathrm{H}), 3.59-3.48(\mathrm{~m}, 3 \mathrm{H}), 2.39(\mathrm{dd}, J=13.2,4.8 \mathrm{~Hz}, 1 \mathrm{H}), 2.32(\mathrm{dd}, J=13.1,3.9$ Hz, $1 \mathrm{H}), 2.20-2.16(\mathrm{~m}, 1 \mathrm{H}), 2.11$ (ddd, $J=14.3,9.0,5.9 \mathrm{~Hz}, 1 \mathrm{H}), 2.07-1.97$ (m, $3 \mathrm{H}), 1.83$ (ddt, $J=14.0,8.7,5.3 \mathrm{~Hz}, 1 \mathrm{H}), 1.77-1.71(\mathrm{~m}, 1 \mathrm{H}), 1.60(\operatorname{app~q}, J=12.1 \mathrm{H}, 1 \mathrm{H}), 1.50(\mathrm{ddd}, J=$ 13.4, 7.5, $5.4 \mathrm{~Hz}, 1 \mathrm{H}), 1.32(\operatorname{app~q}, J=11.2 \mathrm{~Hz}, 1 \mathrm{H}), 1.08-1.06(\mathrm{~m}, 21 \mathrm{H}) ;{ }^{13} \mathrm{C}$ NMR $(125$ $\left.\mathrm{MHz}, \mathrm{CDCl}_{3}\right) \delta 158.8,142.4,141.8,138.5,136.2,128.3,127.5,127.4,110.5,72.9,70.9,68.8$, 68.5, 68.4, 66.7, 41.1, 40.7, 40.0, 39.3, 38.6, 35.8, 34.2, 18.1, 12.3; HRMS (CI/Ammonia) $\mathrm{m} / z$ calcd for $\mathrm{C}_{34} \mathrm{H}_{52} \mathrm{ClNO}_{5} \mathrm{Si}[\mathrm{M}]^{+}$617.3303, found 617.3300.

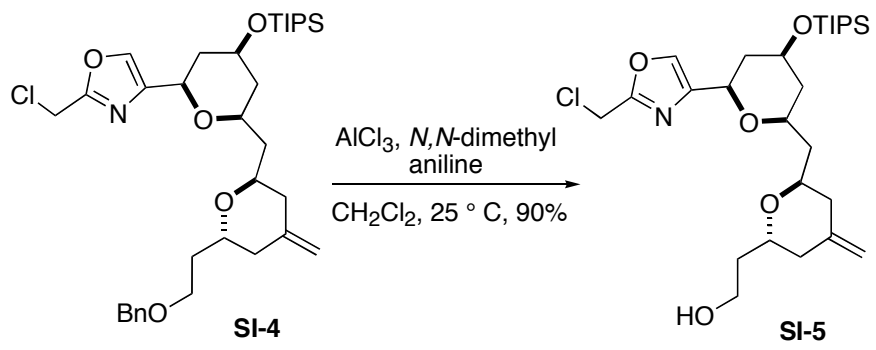

Alcohol SI-5. To a solution of SI-4 $(73.0 \mathrm{mg}, 0.118 \mathrm{mmol}, 1.0$ equiv) in $1 \mathrm{~mL}$ of dichloromethane at $25{ }^{\circ} \mathrm{C}$ was added $N, N$-dimethylaniline (299 $\mu \mathrm{L}, 2.36$ mmol, 20.0 equiv) dropwise via syringe and aluminum trichloride $(252 \mathrm{mg}, 1.89 \mathrm{mmol}, 16.0$ equiv) as a solid in one portion. The reaction mixture was stirred at $25^{\circ} \mathrm{C}$ for 1 hour and then cooled to $0{ }^{\circ} \mathrm{C}$ where upon $1 \mathrm{~N} \mathrm{NaHSO}_{4}(2 \mathrm{~mL})$ was slowly added. The reaction mixture was warmed to $25^{\circ} \mathrm{C}$ and partitioned between ethyl acetate $(20 \mathrm{~mL})$ and saturated aqueous $\mathrm{NaHCO}_{3}(20 \mathrm{~mL})$. The organic layer was then washed with brine $(20 \mathrm{~mL})$, dried over anhydrous $\mathrm{MgSO}_{4}$, filtered and concentrated in vacuo. The resulting crude oil was purified by flash chromatography on silica 
gel (50\% ether/hexanes to 50\% ethyl acetate/hexanes) to afford the desired alcohol (56 mg, 90\%) as a colorless oil: $[\alpha]{ }_{D}^{24}-23.9\left(c \quad 0.410, \mathrm{CH}_{2} \mathrm{Cl}_{2}\right.$ ); IR (thin film) 3428, 2946, 2868, 1463, 1432, 1370, $1096 \mathrm{~cm}^{-1} ;{ }^{1} \mathrm{H} \mathrm{NMR}\left(500 \mathrm{MHz}, \mathrm{CDCl}_{3}\right) \delta 7.59(\mathrm{~d}, J=0.8 \mathrm{~Hz}, 1 \mathrm{H}), 4.77($ br s, $1 \mathrm{H}), 4.73$, (br s, $1 \mathrm{H}), 4.58(\mathrm{~s}, 2 \mathrm{H}), 4.36(\mathrm{dd}, J=11.8,1.2 \mathrm{~Hz}, 1 \mathrm{H}), 4.11-4.06(\mathrm{~m}, 1 \mathrm{H}), 4.05-3.95(\mathrm{~m}, 2$ H), 3.72-3.69 (m, 2 H), 3.58-3.52 (m, 1 H), $2.70($ br s, $1 \mathrm{H}), 2.40$ (dd, $J=13.3,5.0 \mathrm{~Hz}, 1 \mathrm{H})$, $2.28(\mathrm{dd}, J=13.2,3.7 \mathrm{~Hz}, 1 \mathrm{H}), 2.12-2.18(\mathrm{~m}, 1 \mathrm{H}), 2.12(\mathrm{ddd}, J=14.4,9.1,6.4 \mathrm{~Hz}, 1 \mathrm{H}), 2.05-$ $1.98(\mathrm{~m}, 3 \mathrm{H}), 1.84$ (dddd, $J=14.5,9.7,6.9,4.8 \mathrm{~Hz}, 1 \mathrm{H}), 1.68-1.55(\mathrm{~m}, 2 \mathrm{H}), 1.51$ (ddd, $J=$ 14.3, 6.1, $5.3 \mathrm{~Hz}, 1 \mathrm{H}), 1.37$ (app q, $J=11.2 \mathrm{~Hz}, 1 \mathrm{H}), 1.07-1.04(\mathrm{~m}, 21 \mathrm{H}) ;{ }^{13} \mathrm{C}$ NMR $(125 \mathrm{MHz}$, $\left.\mathrm{CDCl}_{3}\right) \delta 159.0,142.2,141.4,136.4,110.7,73.7,71.1,71.0,69.9,68.3,60.7,41.6,40.4,40.1$, 39.1, 38.4, 36.2, 35.8, 18.1, 12.3; HRMS (CI/Ammonia) $\mathrm{m} / \mathrm{z}$ calcd for $\mathrm{C}_{27} \mathrm{H}_{46} \mathrm{ClNO}_{5} \mathrm{Si}[\mathrm{M}-$ $\left.\mathrm{C}_{3} \mathrm{H}_{7}\right]^{+} 484.2286$, found 484.2280 .

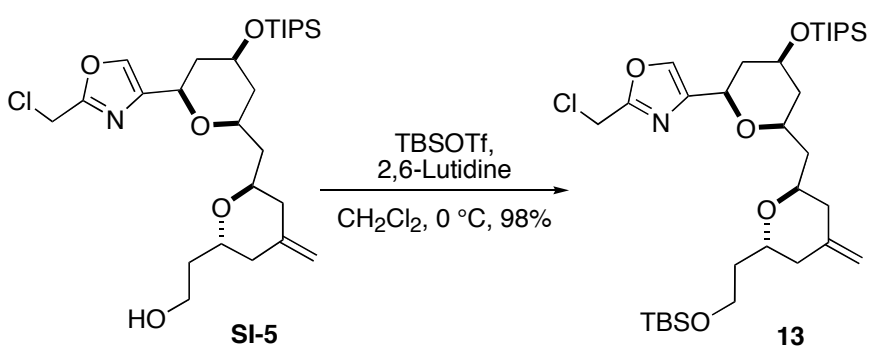

Bis-Tetrahydropyran 13. To a solution of SI-5 $(111 \mathrm{mg}, 0.210 \mathrm{mmol}, 1.0$ equiv) in $2 \mathrm{~mL}$ of dichloromethane at $25{ }^{\circ} \mathrm{C}$ was added 2,6-lutidine $(50 \mu \mathrm{L}, 0.420 \mathrm{mmol}, 2.0$ equiv) dropwise via syringe. The reaction mixture was cooled $0{ }^{\circ} \mathrm{C}$ and TBSOTf $(72.0 \mu \mathrm{L}, 0.315 \mathrm{mmol}, 1.5$ equiv) was added dropwise via syringe. The reaction mixture was stirred at $0{ }^{\circ} \mathrm{C}$ for ten minutes whereupon saturated aqueous $\mathrm{NaHCO}_{3}(2 \mathrm{~mL})$ was added. The reaction mixture was warmed to $25^{\circ} \mathrm{C}$ and diluted with ethyl acetate $(20 \mathrm{~mL})$ and additional saturated aqueous $\mathrm{NaHCO}_{3}(20 \mathrm{~mL})$. 
The organic layer was then washed with $1 \mathrm{~N} \mathrm{NaHSO}_{4}(20 \mathrm{~mL})$, saturated aqueous $\mathrm{NaHCO}_{3}(20$ $\mathrm{mL})$, brine $(20 \mathrm{~mL})$, dried over anhydrous $\mathrm{MgSO}_{4}$, filtered and concentrated in vacuo. The resulting crude oil was purified by flash chromatography on silica gel (5\% ether/hexanes to $50 \%$ ether/hexanes) to afford silyl ether $13(133 \mathrm{mg}, 98 \%)$ as a colorless oil: $[\alpha]{ }_{\mathrm{D}}^{24}-31.7(c 0.6$, $\mathrm{CH}_{2} \mathrm{Cl}_{2}$ ); IR (neat/NaCl) 2945, 2892, 2865, 1463, $1096 \mathrm{~cm}^{-1} ;{ }^{1} \mathrm{H}$ NMR (500 MHz, $\left.\mathrm{CDCl}_{3}\right) \delta 7.57$ (s, $1 \mathrm{H}), 4.76$ (br s, $1 \mathrm{H}), 4.74$ (br s, $1 \mathrm{H}), 4.59$ (s, $2 \mathrm{H}), 4.36$ (d, J = 11.5 Hz, $1 \mathrm{H}), 4.01-3.90$ (m, $2 \mathrm{H}), 3.67-3.64(\mathrm{~m}, 2 \mathrm{H}), 3.59-3.54(\mathrm{~m}, 1 \mathrm{H}), 2.38-2.32(\mathrm{~m}, 2 \mathrm{H}), 2.23(\mathrm{dt}, J=12.6,2.2 \mathrm{~Hz}, 1$ H), 1.80-1.73 (m, $1 \mathrm{H}), 1.63-1.56(\mathrm{~m}, 2 \mathrm{H}), 1.52$ (ddd, $J=13.4,7.2,5.6 \mathrm{~Hz}, 1 \mathrm{H}), 1.32$ (app q, $J$ $=11.5 \mathrm{~Hz}, 1 \mathrm{H}), 1.08-1.05(\mathrm{~m}, 21 \mathrm{H}), 0.89(\mathrm{~s}, 9 \mathrm{H}), 0.05($ br s, $6 \mathrm{H}) ;{ }^{13} \mathrm{C}$ NMR $(125 \mathrm{MHz}$, $\left.\mathrm{CDCl}_{3}\right) \delta 158.9,142.5,142.0,136.2,110.3,73.0,71.1,68.8,68.6,68.4,59.7,41.2,40.8,39.8$, 39.5, 39.2, 36.8, 35.8, 25.9, 18.3, 18.1, 12.3, -5.3; HRMS (CI/Ammonia) $\mathrm{m} / \mathrm{z}$ calcd for $\mathrm{C}_{33} \mathrm{H}_{60} \mathrm{ClNO}_{5} \mathrm{Si}_{2}\left[\mathrm{M}-\mathrm{C}_{3} \mathrm{H}_{7}\right]^{+}$598.3151, found 598.3142.

\section{Synthesis of Product 24 using a TMSBr-Promoted Cyclization}
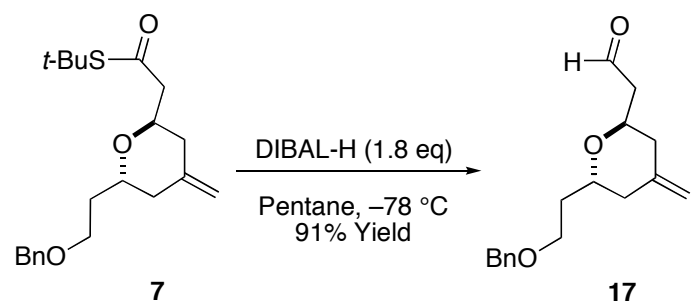

Aldehyde 17. To a cooled $\left(-78^{\circ} \mathrm{C}\right)$ solution of thioester $7(1.13 \mathrm{~g}, 3.12 \mathrm{mmol}, 1.0 \mathrm{eq})$ in $30 \mathrm{~mL}$ of dichloromethane was added DIBAL-H (5.62 mL, 1.0 M in toluene, $5.62 \mathrm{mmol}, 1.8 \mathrm{eq})$ over 10 minutes. After stirring at $-78{ }^{\circ} \mathrm{C}$ for 15 minutes, the reaction was quenched by dropwise addition of ethyl formate $(1 \mathrm{~mL})$. The reaction mixture was then diluted with saturated aqueous sodium potassium tartrate $(50 \mathrm{~mL})$, warmed to $25^{\circ} \mathrm{C}$ and stirred vigorously for 2 hours. The 
reaction mixture was then diluted with $50 \mathrm{~mL}$ of $\mathrm{H}_{2} \mathrm{O}$, and the aqueous layer was extracted with dichloromethane $\left(3 \times 25 \mathrm{~mL}\right.$ ). The combined organic layers were dried over $\mathrm{Na}_{2} \mathrm{SO}_{4}$, filtered and concentrated in vacuo. The resulting crude oil was purified by flash chromatography on silica gel (10\% ether/hexanes to 50\% ether/hexanes) to afford aldehyde 17 (776 mg, 91\%) and recovered starting material $(106 \mathrm{mg}, 9 \%)$ both as colorless oils. Data for $\mathbf{1 7}$ is presented: $\left[\alpha{ }^{24}{ }_{\mathrm{D}}\right.$ -54.1 ( $c$ 0.315, $\mathrm{CHCl}_{3}$ ); IR (neat/NaCl) 2943, 2860, 1724, 1366, 1099, $1031 \mathrm{~cm}^{-1} ;{ }^{1} \mathrm{H}$ NMR (500 $\left.\mathrm{MHz}, \mathrm{CDCl}_{3}\right) \delta 9.73(\mathrm{dd}, J=2.6,1.8 \mathrm{~Hz}, 1 \mathrm{H}), 7.36-7.32(\mathrm{~m}, 3 \mathrm{H}), 7.29-7.27(\mathrm{~m}, 2 \mathrm{H}), 4.80$ (br s, $1 \mathrm{H}), 4.79($ br s, $1 \mathrm{H}), 4.49(\mathrm{ABq}, J=11.9 \mathrm{~Hz}, \Delta v=9.5 \mathrm{~Hz}, 2 \mathrm{H}), 4.40-4.35(\mathrm{~m}, 1 \mathrm{H}), 4.00-$ $3.95(\mathrm{~m}, 1 \mathrm{H}), 3.54-3.50(\mathrm{~m}, 2 \mathrm{H}), 4.72(\mathrm{ddd}, J=16.3,8.4,2.8 \mathrm{~Hz}, 1 \mathrm{H}), 2.46(\mathrm{ddd}, J=16.2,5.2$, $1.7 \mathrm{~Hz}, 1 \mathrm{H}), 2.41(\mathrm{dd}, J=13.3,4.6 \mathrm{~Hz}, 1 \mathrm{H}), 2.36(\mathrm{dd}, J=13.3,4.3 \mathrm{~Hz}, 1 \mathrm{H}), 2.05-2.00$ (m, 2 H), 1.92 (dddd, $J=14.5,9.2,5.5,5.5 \mathrm{~Hz}, 1 \mathrm{H}), 1.73-1.67(\mathrm{~m}, 1 \mathrm{H}) ;{ }^{13} \mathrm{C}$ NMR $\left(125 \mathrm{MHz}, \mathrm{CDCl}_{3}\right)$ б 201.3, 141.4, 138.9, 128.8, 128.2, 128.0, 111.7, 73.6, 70.1, 68.0, 67.1, 47.6, 40.0, 39.8, 33.9; HRMS (CI/Ammonia) $m / z$ calcd for $\mathrm{C}_{17} \mathrm{H}_{22} \mathrm{O}_{3}[\mathrm{M}+\mathrm{H}]^{+} 275.1647$, found 275.1651.

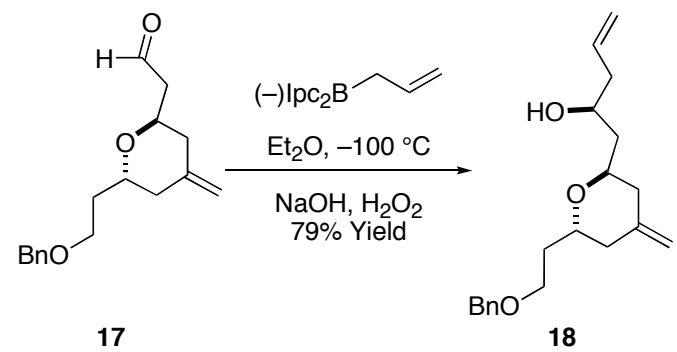

Homoallylic Alcohol 18. The general procedure for the asymmetric allylation of aldehydes developed by Brown was followed. ${ }^{10}$ To a cooled $\left(-110{ }^{\circ} \mathrm{C}\right)$ solution of $(-)-B-$ allyldiisopinocampheylborane (19.6 mL, salt-free 0.20 M in diethyl ether, $3.93 \mathrm{mmol}, 1.5$ equiv)

\footnotetext{
${ }^{10}$ Jadhav, P.K.; Bhat, K.S.; Perumal, P.T.; Brown, H.C. J. Org. Chem. 1986, 51, 432-439.
} 
was added a cooled $\left(-78{ }^{\circ} \mathrm{C}\right)$ solution of aldehyde $17(720 \mathrm{mg}, 2.62 \mathrm{mmol}, 1.0 \mathrm{eq})$ in $5 \mathrm{~mL}$ of diethyl ether dropwise over 15 minutes. The reaction was warmed to $-100{ }^{\circ} \mathrm{C}$ over 20 minutes, stirred for an additional 15 minutes and then quenched by dropwise addition of methanol ( $5 \mathrm{~mL})$. The reaction was warmed to $0{ }^{\circ} \mathrm{C}$, treated with $1 \mathrm{~N} \mathrm{NaOH}(5 \mathrm{~mL})$ and $30 \% \mathrm{H}_{2} \mathrm{O}_{2}(5 \mathrm{~mL})$ and stirred vigorously for 1.5 hours. The reaction mixture was diluted with brine $(20 \mathrm{~mL})$ and extracted with ethyl acetate $(4 \times 15 \mathrm{~mL})$. The combined organic extracts were washed with brine (15 mL), dried over $\mathrm{Na}_{2} \mathrm{SO}_{4}$, filtered and concentrated in vacuo. The resulting crude oil was purified by flash chromatography on silica gel (20\% ethyl acetate/hexanes to $50 \%$ ethyl acetate/hexanes) to afford the title homoallylic alcohol contaminated with isopinocampheol. Vacuum drying $\left(0.1\right.$ Torr, $\left.60{ }^{\circ} \mathrm{C}, 18 \mathrm{~h}\right)$ removed the residual isopinocampheol and afforded 18 (654 mg, 79\%) as a colorless oil: $[\alpha]_{\mathrm{D}}^{24}-56.8\left(c\right.$ 1.15, $\mathrm{CH}_{2} \mathrm{Cl}_{2}$ ); IR (thin film) 3476, 3074, 2943, 2866, 1653, $1097 \mathrm{~cm}^{-1} ;{ }^{1} \mathrm{H}$ NMR (500 MHz, $\mathrm{CDCl}_{3}$ ) $\delta$ 7.35-7.32 (m, 3 H), 7.30-7.27 (m, $\left.2 \mathrm{H}\right)$, $5.82(\mathrm{ddt}, J=17.3,10.3,7.1 \mathrm{~Hz}, 1 \mathrm{H}), 5.11-5.05$ (m, $2 \mathrm{H}), 4.78$ (br s, $1 \mathrm{H}), 4.74$ (br s, $1 \mathrm{H}), 4.50$ $(\mathrm{ABq}, J=11.8 \mathrm{~Hz}, \Delta v=23.2 \mathrm{~Hz}, 2 \mathrm{H}), 4.17$ (app sextet, $J=4.7 \mathrm{~Hz}, 1 \mathrm{H}), 3.39-3.88(\mathrm{~m}, 1 \mathrm{H})$, 3.82-3.77 (m, $1 \mathrm{H}), 3.58-3.51(\mathrm{~m}, 3 \mathrm{H}), 2.43(\mathrm{dd}, J=13.3,5.3 \mathrm{~Hz}, 1 \mathrm{H}), 2.28-2.17(\mathrm{~m}, 3 \mathrm{H})$, 2.06-2.00 (m, $3 \mathrm{H}), 1.72-1.64(\mathrm{~m}, 2 \mathrm{H}), 1.53(\mathrm{dt}, J=14.5,2.7 \mathrm{~Hz}, 1 \mathrm{H}) ;{ }^{13} \mathrm{C} \mathrm{NMR}(125 \mathrm{MHz}$, $\left.\mathrm{CDCl}_{3}\right) \delta 141.2,138.3,134.4,128.3,127.7,127.5,117.3,110.6,73.1,72.3,71.0,70.4,66.8$, 41.9, 40.7, 40.5, 38.9, 32.0; HRMS (CI/Ammonia) $m / z$ calcd for $\mathrm{C}_{20} \mathrm{H}_{28} \mathrm{O}_{3}[\mathrm{M}+\mathrm{H}]^{+} 317.2116$, found 317.2112. 


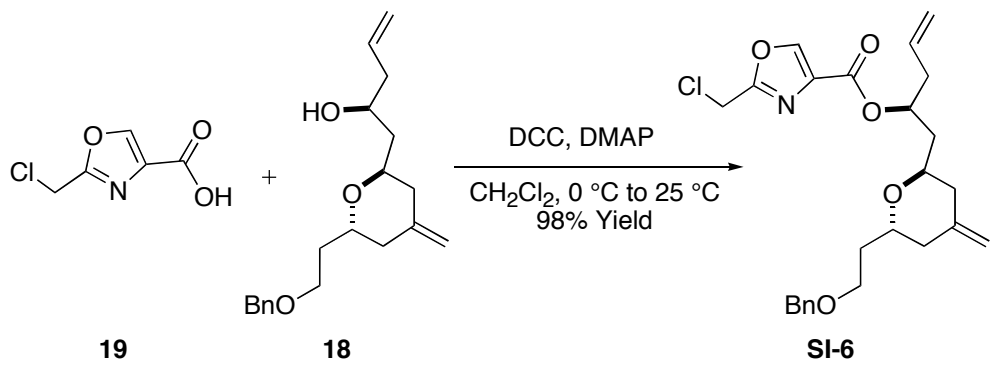

Ester SI-6. To a cooled $\left(0{ }^{\circ} \mathrm{C}\right)$ solution of carboxylic acid $\mathbf{1 9}^{11}(166 \mathrm{mg}, 1.03 \mathrm{mmol}, 1.3$ equiv) and homoallylic alcohol 18 (250 mg, $0.790 \mathrm{mmol}, 1.0$ equiv) in $7 \mathrm{~mL}$ of dichloromethane was added dicyclohexylcarbodiimide $\quad(179 \mathrm{mg}, \quad 0.869 \mathrm{mmol}, \quad 1.1$ equiv $)$ and $\quad N, N-$ dimethylaminopyridine ( $19.0 \mathrm{mg}, 0.158 \mathrm{mmol}, 0.2$ equiv). The reaction mixture was warmed to $25^{\circ} \mathrm{C}$, stirred for 12 hours, diluted with pentane $(20 \mathrm{~mL})$ and filtered to remove the precipitated dicylohexylurea. The resulting solution was concentrated in vacuo to provide a crude oil that was purified by flash chromatography on silica gel (10\% ether/hexanes to $60 \%$ ether/hexanes) affording SI-6 (356 mg, 98\%) as a colorless oil: $[\alpha]{ }_{D}^{24}-18.6\left(c 1.23, \mathrm{CH}_{2} \mathrm{Cl}_{2}\right)$; IR (thin film) $3159,3035,2943,2866,1738,1104 \mathrm{~cm}^{-1} ;{ }^{1} \mathrm{H}$ NMR $\left(500 \mathrm{MHz}, \mathrm{C}_{6} \mathrm{D}_{6}\right) \delta 7.49(\mathrm{~s}, 1 \mathrm{H}), 7.35(\mathrm{~d}, J=$ $7.3 \mathrm{~Hz}, 2 \mathrm{H}), 7.20$ (t, $J=7.6 \mathrm{~Hz}, 2 \mathrm{H}), 7.11-7.10(\mathrm{~m}, 1 \mathrm{H}), 5.81$ (ddt, $J=17.0,10.2,6.6 \mathrm{~Hz}, 1$ H), 5.46-5.41 (m, $1 \mathrm{H}), 5.06-4.99(\mathrm{~m}, 2 \mathrm{H}), 4.70(\mathrm{~s}, 1 \mathrm{H}), 4.69(\mathrm{~s}, 1 \mathrm{H}), 4.45(\mathrm{~d}, J=12.1 \mathrm{~Hz}, 1$ H), $4.38(\mathrm{~d}, J=12.1 \mathrm{~Hz}, 1 \mathrm{H}), 4.04-3.99(\mathrm{~m}, 1 \mathrm{H}), 3.93-3.89(\mathrm{~m}, 1 \mathrm{H}), 3.79(\mathrm{~s}, 2 \mathrm{H}), 3.59-3.55$ (m, $1 \mathrm{H}), 3.52-3.49(\mathrm{~m}, 1 \mathrm{H}), 2.44-2.32(\mathrm{~m}, 2 \mathrm{H}), 2.24(\mathrm{dd}, J=13.2,4.7 \mathrm{~Hz}, 1 \mathrm{H}), 2.19-2.13(\mathrm{~m}$, $2 \mathrm{H}), 1.90$ (ddt, $J=14.1,8.5,5.7 \mathrm{~Hz}, 1 \mathrm{H}), 1.85(\mathrm{dd}, J=13.1,5.9 \mathrm{~Hz}, 2 \mathrm{H}), 1.70-1.64(\mathrm{~m}, 1 \mathrm{H})$, $1.55(\mathrm{ddd}, J=14.1,6.7,5.4 \mathrm{~Hz}, 1 \mathrm{H}) ;{ }^{13} \mathrm{C} \mathrm{NMR}\left(125 \mathrm{MHz}, \mathrm{CDCl}_{3}\right) \delta 160.1,159.7,144.9,141.4$, 138.5, 134.0, 133.1, 128.3, 127.6, 127.4, 118.3, 110.7, 73.0, 72.0, 69.1(2), 66.9, 39.9, 39.1, 38.1,

\footnotetext{
${ }^{11}$ Breuilles, P.; Uguen, D. Tetrahedron Lett. 1998, 39, 3149-3152.
} 
36.4, 35.3, 34.1; HRMS (CI/Ammonia) $\mathrm{m} / z$ calcd for $\mathrm{C}_{25} \mathrm{H}_{30} \mathrm{ClNO}_{5}[\mathrm{M}]^{+}$459.1812, found 459.1812.

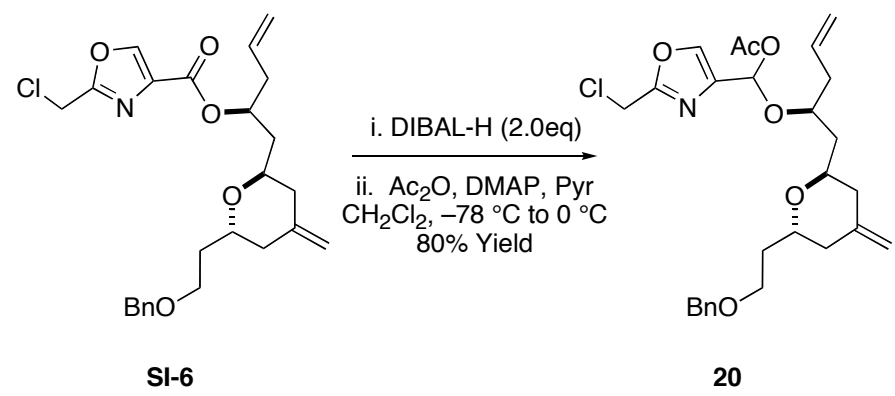

Acetoxy Ether 20. DIBAL-H reduction/in situ acetylation of ester SI-6 (50 mg, $0.109 \mathrm{mmol}$, 1.00 equiv) according to the general procedure for the preparation of $\alpha$-acetoxy ethers provided crude acetoxy ether 20. ${ }^{12}$ While this material was generally taken on to the next step without further purification, an analytical sample was obtained by flash chromatography on silica gel deactivated with $2 \%$ triethylamine (10\% ether/hexanes to $50 \%$ ether/hexanes) to afford 20 (43.6 mg, 80\%) as a colorless oil: IR (thin film) 3074, 2943, 2866, 1746, 1375, 1236, $1097 \mathrm{~cm}^{-1} ;{ }^{1} \mathrm{H}$ NMR (500 MHz, $\left.\mathrm{CDCl}_{3}\right) \delta 7.68(\operatorname{app~t}, J=1.1 \mathrm{~Hz}, 1 \mathrm{H}), 7.35-7.31(\mathrm{~m}, 3 \mathrm{H}), 7.29-7.25$ (m, $\left.2 \mathrm{H}\right)$, $6.80(\mathrm{~d}, J=0.8 \mathrm{~Hz}, 0.5 \mathrm{H}), 6.79(\mathrm{~d}, J=0.7 \mathrm{~Hz}, 0.5 \mathrm{H}), 5.89-5.75(\mathrm{~m}, 1 \mathrm{H}), 5.11-5.06(\mathrm{~m}, 2 \mathrm{H})$, 4.76-4.74 (m, $2 \mathrm{H}), 4.58$ (s, $1 \mathrm{H}), 4.56$ (s, $1 \mathrm{H}), 4.53-4.46$ (m, $2 \mathrm{H}), 3.97-3.85$ (m, $3 \mathrm{H}), 3.55-$ $3.50(\mathrm{~m}, 2 \mathrm{H}), 2.44-2.27(\mathrm{~m}, 4 \mathrm{H}), 2.12(\mathrm{~s}, 1.5 \mathrm{H}), 2.11(\mathrm{~s}, 1.5 \mathrm{H}), 2.01-1.94(\mathrm{~m}, 2 \mathrm{H}), 1.93-1.85$ $(\mathrm{m}, 1 \mathrm{H}), 1.74-1.66(\mathrm{~m}, 2 \mathrm{H}), 1.65-1.58(\mathrm{~m}, 1 \mathrm{H}) ;{ }^{13} \mathrm{C} \mathrm{NMR}\left(125 \mathrm{MHz}, \mathrm{CDCl}_{3}\right) \delta$ 170.4, 170.2, $159.5,159.4,141.8,141.6,139.2,139.1,138.5,138.0,137.9,134.2,133.5,128.3,127.6$, 127.5(2), 118.2, 117.4, 110.6, 110.5, 90.8(2), 77.3, 76.3, 73.0, 69.3, 69.2, 68.7(2), 66.9(2), 39.9,

\footnotetext{
${ }^{12}$ For the preparation of $\alpha$-acetoxy ethers, see: (a) Kopecky, D.J.; Rychnovsky, S.D. J. Org.
} Chem. 2000, 65, 191-198. (b) Kopecky, D.J.; Rychnovsky, S.D. Org. Synth. 2003, 80, 177-183. 
39.6, 39.0, 38.8, 38.6, 37.6, 35.6(2), 33.9, 33.6, 21.2(2); HRMS (CI/Ammonia) $\mathrm{m} / \mathrm{z}$ calcd for $\mathrm{C}_{27} \mathrm{H}_{34} \mathrm{ClNO}_{6}[\mathrm{M}+\mathrm{Na}]^{+}$526.1973, found 526.1970. 


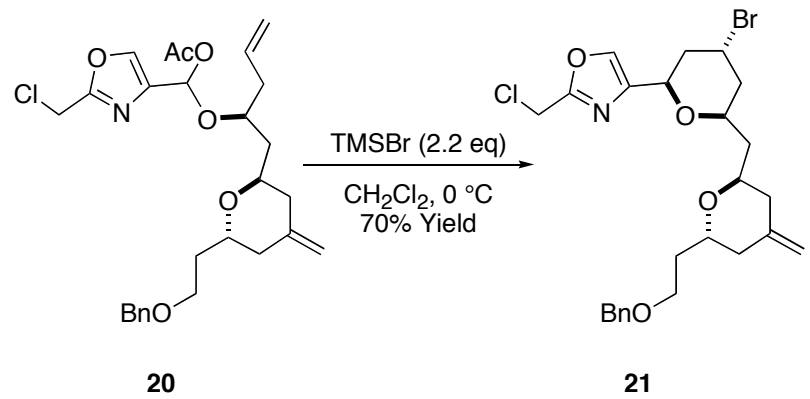

Bromotetrahydropyran 21 . To a cooled $\left(0{ }^{\circ} \mathrm{C}\right)$ solution of acetoxy acetal $20(85.0 \mathrm{mg}, 0.169$ mmol, 1.0 equiv) in $1.5 \mathrm{~mL}$ of dichloromethane was added $\mathrm{TMSBr}(49.0 \mu \mathrm{L}, 0.371 \mathrm{mmol}, 2.2$ equiv) dropwise via syringe. The reaction mixture was stirred at $0{ }^{\circ} \mathrm{C}$ for 3 hours and was then diluted with saturated aqueous $\mathrm{NaHCO}_{3}(10 \mathrm{~mL})$ and ether $(10 \mathrm{~mL})$. After warming to $25{ }^{\circ} \mathrm{C}$ and stirring for 20 minutes, the layers were separated, and the organic phase was dried over anhydrous $\mathrm{MgSO}_{4}$, filtered and concentrated in vacuo. The resulting crude oil was purified by flash chromatography on silica gel (10\% ether/hexanes to $60 \%$ ether/hexanes) to afford $\mathbf{2 1}(62.0$ mg, 70\%) as a colorless oil: $[\alpha]{ }_{\mathrm{D}}^{24}-26.7$ (c 1.21, $\mathrm{CH}_{2} \mathrm{Cl}_{2}$ ); IR (thin film) 3035, 2950, 2866, 1576, $1074 \mathrm{~cm}^{-1} ;{ }^{1} \mathrm{H}$ NMR (500 MHz, $\left.\mathrm{CDCl}_{3}\right) \delta 7.50$ (d, $\left.J=0.8 \mathrm{~Hz}, 1 \mathrm{H}\right), 7.35-7.31(\mathrm{~m}, 3 \mathrm{H})$, 7.28-7.24 (m, $2 \mathrm{H}), 4.91(\mathrm{dd}, J=10.7,2.3 \mathrm{~Hz}, 1 \mathrm{H}), 4.81(\mathrm{~m}, 1 \mathrm{H}), 4.76$ (br s, $1 \mathrm{H}), 4.74$ (br s, 1 H), $4.58(\mathrm{~d}, J=12.2 \mathrm{~Hz}, 1 \mathrm{H}), 4.57(\mathrm{~s}, 2 \mathrm{H}), 4.51(\mathrm{~d}, J=12.0 \mathrm{~Hz}, 1 \mathrm{H}), 4.17-4.12(\mathrm{~m}, 1 \mathrm{H})$, 4.00-3.94 (m, $2 \mathrm{H}), 3.66-3.61(\mathrm{~m}, 1 \mathrm{H}), 3.59-3.55(\mathrm{~m}, 1 \mathrm{H}), 2.38$ (dd, $J=13.2,4.7 \mathrm{~Hz}, 1 \mathrm{H})$, $2.33(\mathrm{dd}, J=13.2,3.9 \mathrm{~Hz}, 1 \mathrm{H}), 2.27-2.23(\mathrm{~m}, 1 \mathrm{H}), 2.19(\mathrm{dd}, J=10.9,3.3 \mathrm{~Hz}, 1 \mathrm{H}), 2.17-2.09$ (m, 2 H), 2.04-1.97 (m, 2 H), 1.92-1.80 (m, 2 H), 1.74-1.67 (m, 1 H), 1.49 (ddd, J = 13.0, 7.8, $5.1 \mathrm{~Hz}, 1 \mathrm{H}) ;{ }^{13} \mathrm{C} \mathrm{NMR}\left(125 \mathrm{MHz}, \mathrm{CDCl}_{3}\right) \delta 159.4,143.2,142.9,139.9,137.0,128.9,128.0$, $127.9,110.7,73.5,70.2,69.3,68.9,68.8,67.6,50.6,40.7,40.2,39.4(2), 39.2,36.1,34.9$; HRMS (CI/Ammonia) $m / z$ calcd for $\mathrm{C}_{25} \mathrm{H}_{31} \mathrm{BrClNO}_{4}[\mathrm{M}]^{+}$523.1125, found 523.1122. 


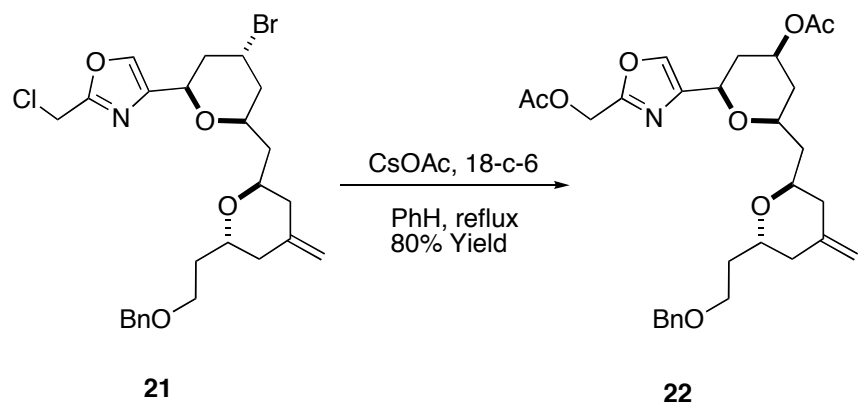

Bis-Acetate 22. To a solution of bromotetrahydropyran $21(62.0 \mathrm{mg}, 0.118 \mathrm{mmol}, 1.0$ equiv) in $1 \mathrm{~mL}$ of toluene at $25^{\circ} \mathrm{C}$ was added $18-\mathrm{C}-6$ (31.0 mg, $0.118 \mathrm{mmol}, 1.0$ equiv) and CsOAc (159 $\mathrm{mg}, 0.827 \mathrm{mmol}, 7.0$ equiv). The resulting suspension was heated to $85^{\circ} \mathrm{C}$ and stirred for 48 hours. After cooling to $25^{\circ} \mathrm{C}$, the suspension was partitioned between half-saturated aqueous $\mathrm{NaHCO}_{3}(10 \mathrm{~mL})$ and ether $(10 \mathrm{~mL})$. The aqueous layer was extracted with ether $(3 \times 10 \mathrm{~mL})$, and the combined organic extracts were dried over anhydrous $\mathrm{MgSO}_{4}$, filtered and concentrated in vacuo. The resulting crude oil was purified by flash chromatography on silica gel $(10 \%$ ether/hexanes to $100 \%$ ether) to afford $22(50.0 \mathrm{mg}, 80 \%)$ as a colorless oil: $[\alpha]{ }_{\mathrm{D}}^{24}-33.8(\mathrm{c}$ $0.715, \mathrm{CH}_{2} \mathrm{Cl}_{2}$ ); IR (thin film) 2926, 2856, 1745, 1366, 1235, 1096, $1031 \mathrm{~cm}^{-1} ;{ }^{1} \mathrm{H}$ NMR $(500$ $\left.\mathrm{MHz}, \mathrm{CDCl}_{3}\right) \delta 7.48(\mathrm{~d}, J=1.0 \mathrm{~Hz}, 1 \mathrm{H}), 7.36-7.31(\mathrm{~m}, 3 \mathrm{H}), 7.28-7.24(\mathrm{~m}, 2 \mathrm{H}), 5.11(\mathrm{~s}, 2 \mathrm{H})$, 4.97 (tt, $J=11.2,4.7 \mathrm{~Hz}, 1 \mathrm{H}), 4.75$ (br s, $1 \mathrm{H}), 4.73$ (br s, $1 \mathrm{H}), 4.51$ (s, $2 \mathrm{H}), 4.29$ (dd, $J=12.0$, $1.0 \mathrm{~Hz}, 1 \mathrm{H}), 4.01-3.96$ (m, $1 \mathrm{H}), 3.96-3.91$ (m, $1 \mathrm{H}), 3.63-3.55$ (m, $2 \mathrm{H}), 3.53-3.49$ (m, $1 \mathrm{H})$, $2.36(\mathrm{dd}, J=13.2,4.7 \mathrm{~Hz}, 1 \mathrm{H}), 2.31(\mathrm{dd}, J=13.2,3.9 \mathrm{~Hz}, 1 \mathrm{H}), 2.26-2.23(\mathrm{~m}, 1 \mathrm{H}), 2.13-2.07$ (m, 2 H), 2.12 (s, 3 H), 2.04 (s, 3 H), 2.01-1.95 (m, 2 H), 1.84 (dddd, $J=14.2,8.9,5.3,5.3 \mathrm{~Hz}, 1$ H), 1.75-1.65 (m, $2 \mathrm{H}), 1.52$ (ddd, $J=13.2,7.4,5.5 \mathrm{~Hz}, 1 \mathrm{H}), 1.38$ (app q, $J=11.5 \mathrm{~Hz}, 1 \mathrm{H}) ;{ }^{13} \mathrm{C}$ NMR (125 MHz, $\left.\mathrm{CDCl}_{3}\right) \delta 170.3,170.1,158.7,141.7,141.5,138.5,136.0,128.3,127.5(2)$, 
110.6, 73.0, 72.9, 70.6, 69.9, 68.7, 68.5, 66.8, 57.7, 40.0, 39.3, 38.6, 36.5, 36.1, 34.1, 21.2, 20.5;

HRMS (CI/Ammonia) $m / z$ calcd for $\mathrm{C}_{29} \mathrm{H}_{37} \mathrm{NO}_{8}[\mathrm{M}]^{+}$527.2519, found 527.2509.

\section{Synthesis of Product 13 using a TFA-Promoted Cyclization}
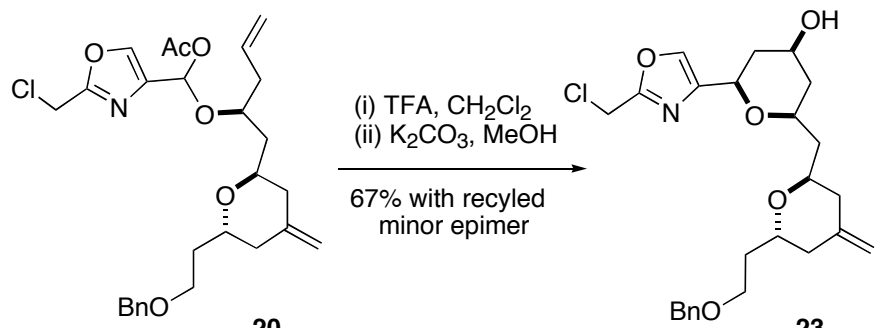

Alcohol 23. To trifluoroacetic acid (10.9 mL, $1.42 \mathrm{mmol}, 1.0$ equiv) at $0{ }^{\circ} \mathrm{C}$ was added a solution of 20 (0.716 g, $1.42 \mathrm{mmol}, 1.0$ equiv) in $\mathrm{CH}_{2} \mathrm{Cl}_{2}(5.0 \mathrm{~mL}, 2.5 \mathrm{~mL}$ rinse $)$ over 5 minutes. After stirring for 15 minutes at $0{ }^{\circ} \mathrm{C}$, the reaction mixture was diluted with $\mathrm{CH}_{2} \mathrm{Cl}_{2}(50 \mathrm{~mL})$ and saturated aqueous $\mathrm{NaHCO}_{3}(50 \mathrm{~mL})$ was added over 15 min. Following separation of the phases, the aqueous layer was extracted with $\mathrm{CH}_{2} \mathrm{Cl}_{2}(2 \times 15 \mathrm{~mL})$. The combined organic phases were then washed with brine $(15 \mathrm{~mL})$, dried over $\mathrm{Na}_{2} \mathrm{SO}_{4}$, filtered and concentrated in vacuo. The resulting pale yellow oil was dissolved in $\mathrm{MeOH}(15 \mathrm{~mL})$, cooled to $0{ }^{\circ} \mathrm{C}$ and treated with $\mathrm{K}_{2} \mathrm{CO}_{3}\left(0.196 \mathrm{mg}, 1.42 \mathrm{mmol}, 1.0\right.$ equiv). After stirring for 10 minutes at $0{ }^{\circ} \mathrm{C}$, water $(50 \mathrm{~mL})$ and EtOAc $(15 \mathrm{~mL})$ were added. Following separation of the phases, the aqueous layer was extracted with EtOAc $(2 \times 15 \mathrm{~mL})$. The combined organic phases were then washed with brine (10 mL), dried over $\mathrm{Na}_{2} \mathrm{SO}_{4}$, filtered and concentrated in vacuo. The resulting crude oil was purified by flash chromatography on silica gel (5\% EtOAc/hexanes to $50 \%$ EtOAc/hexanes) alcohol $23(0.361 \mathrm{~g}, 55 \%)$ and the corresponding axial epimer $(0.098 \mathrm{~g}, 15 \%)$ as colorless oils. 